\title{
THE SPATIALLY RESOLVED STAR FORMATION LAW FROM INTEGRAL FIELD SPECTROSCOPY: VIRUS-P OBSERVATIONS OF NGC 5194
}

\author{
Guillermo A. Blanc, Amanda Heiderman, Karl Gebhardt, Neal J. Evans II, and Joshua Adams \\ Astronomy Department, University of Texas at Austin, Austin, TX 78712, USA \\ Received 2009 June 4; accepted 2009 August 31; published 2009 September 25
}

\begin{abstract}
We investigate the relation between the star formation rate (SFR) surface density $\left(\Sigma_{\mathrm{SFR}}\right)$ and the mass surface density of gas $\left(\Sigma_{\text {gas }}\right)$ in NGC 5194 (a.k.a. M51a, Whirlpool Galaxy). Visible Integral field Replicable Unit Spectrograph Prototype (VIRUS-P) integral field spectroscopy of the central $4.1 \times 4.1 \mathrm{kpc}^{2}$ of the galaxy is used to measure $\mathrm{H} \alpha, \mathrm{H} \beta,[\mathrm{O}$ III] $] 25007,[\mathrm{~N}$ II $] \lambda \lambda 6548,6584$, and $\left[\mathrm{S}_{\mathrm{II}}\right] \lambda \lambda 6717,6731$ emission line fluxes for 735 regions $\sim 170 \mathrm{pc}$ in diameter. We use the Balmer decrement to calculate nebular dust extinctions, and correct the observed fluxes in order to accurately measure $\Sigma_{\mathrm{SFR}}$ in each region. Archival H I $21 \mathrm{~cm}$ and CO maps with spatial resolution similar to that of VIRUS-P are used to measure the atomic and molecular gas surface density for each region. We present a new method for fitting the star formation law (SFL), which includes the intrinsic scatter in the relation as a free parameter, allows the inclusion of non-detections in both $\Sigma_{\text {gas }}$ and $\Sigma_{\mathrm{SFR}}$, and is free of the systematics involved in performing linear regressions over incomplete data in logarithmic space. After rejecting regions whose nebular spectrum is affected by the central active galactic nucleus in NGC 5194, we use the $[\mathrm{SII}] / \mathrm{H} \alpha$ ratio to separate spectroscopically the contribution from the diffuse ionized gas (DIG) in the galaxy, which has a different temperature and ionization state from those of $\mathrm{H}$ II regions in the disk. The DIG only accounts for $11 \%$ of the total $\mathrm{H} \alpha$ luminosity integrated over the whole central region, but on local scales it can account for up to a $100 \%$ of the $\mathrm{H} \alpha$ emission, especially in the interarm regions. After removing the DIG contribution from the $\mathrm{H} \alpha$ fluxes, we measure a slope $N=0.82 \pm 0.05$, and an intrinsic scatter $\epsilon=0.43 \pm 0.02$ dex for the molecular gas SFL. We also measure a typical depletion timescale $\tau=\Sigma_{\mathrm{H}+\mathrm{H}_{2}} / \Sigma_{\mathrm{SFR}} \approx 2 \mathrm{Gyr}$, in good agreement with recent measurements by Bigiel et al. The atomic gas density shows no correlation with the SFR, and the total gas SFL in the sampled density range closely follows the molecular gas SFL. Integral field spectroscopy allows a much cleaner measurement of $\mathrm{H} \alpha$ emission line fluxes than narrow-band imaging, since it is free of the systematics introduced by continuum subtraction, underlying photospheric absorption, and contamination by the [N II] doublet. We assess the validity of different corrections usually applied in narrow-band measurements to overcome these issues and find that while systematics are introduced by these corrections, they are only dominant in the low surface brightness regime. The disagreement with the previous measurement of a super-linear molecular SFL by Kennicutt et al. is most likely due to differences in the fitting method. Our results support the recent evidence for a low, and close to constant, star formation efficiency (SFE $=\tau^{-1}$ ) in the molecular component of the interstellar medium. The data show an excellent agreement with the recently proposed model of the SFL by Krumholz et al. The large intrinsic scatter observed may imply the existence of other parameters, beyond the availability of gas, which are important in setting the SFR.
\end{abstract}

Key words: galaxies: formation - galaxies: individual (NGC 5194) - galaxies: ISM - galaxies: spiral - ISM: structure - stars: formation

Online-only material: color figures, machine-readable table

\section{INTRODUCTION}

In the quest to achieve a thorough understanding of the processes involved in the formation and subsequent evolution of galaxies, we must first fully characterize the process of star formation under different environments in the interstellar medium (ISM). During the last decade, major efforts have been made to characterize the variables involved in triggering star formation and setting the star formation rate (SFR) in galaxies. Kennicutt (1998b) showed that, integrating over the whole optical disk of galaxies, the SFR surface density $\left(\Sigma_{\mathrm{SFR}}\right)$, as measured by the $\mathrm{H} \alpha$ emission, tightly correlates with the total gas surface density $\left(\Sigma_{\mathrm{H}_{\mathrm{I}} \mathrm{H}_{2}}\right)$ over several orders of magnitude in SFR and gas density. The relation from Kennicutt follows a power-law form, with a slope $N=1.4$. These types of correlations between $\Sigma_{\mathrm{SFR}}$ and $\Sigma_{\text {gas }}$, either atomic $\left(\Sigma_{\mathrm{H}_{\mathrm{I}}}\right)$, molecular $\left(\Sigma_{\mathrm{H}_{2}}\right)$, or total $\left(\Sigma_{\mathrm{H}_{\mathrm{I}} \mathrm{H}_{2}}\right)$, are usually known as star formation laws (SFLs, a.k.a. Schmidt Laws or Schmidt-Kennicutt Laws, after Schmidt 1959, who first introduced the power-law parametrization to relate gas density and the SFR), and they show that the availability of gas is a key variable in setting the SFR.

Although the global SFL provides us with valuable insights on the role that gas density plays at setting the SFR, the measurement involves averaging over the many orders of magnitude in $\Sigma_{\text {gas }}$ and $\Sigma_{\text {SFR }}$ present in the ISM of single galaxies, implying the loss of valuable information about the detailed physics that give rise to the SFL. Azimuthally averaged measurements of gas surface densities and the SFR have been used to conduct more detailed studies of the SFL across the disks of local galaxies. For example, Wong \& Blitz (2002) measured, under the assumption of constant dust extinction, a slope of $N \approx 0.8$ for the molecular SFL, and $N \approx 1.1$ for the total gas SFL on a sample of seven molecule rich spirals, with a large scatter from galaxy to galaxy, and Schuster et al. (2007) measured $N=1.4 \pm 0.6$ for the total gas SFL on NGC 5194 . Azimuthally averaged profiles are also affected by averaging 
effects since $\Sigma_{\text {SFR }}$ and $\Sigma_{\text {gas }}$ can change by more than 2 orders of magnitude at constant galactocentric radius due to the presence of spiral structure. We refer the reader to Bigiel et al. (2008) for a thorough compilation of previous measurements of the SFL in local galaxies.

More recently two studies have been aimed at measuring the "spatially resolved" SFL throughout the disks of nearby galaxies. Kennicutt et al. (2007) used a combination of narrowband $\mathrm{H} \alpha$ and $24 \mu \mathrm{m}$ photometry to estimate $\Sigma_{\mathrm{SFR}}$, as well as $21 \mathrm{~cm}$ and CO $J=1-0$ maps to measure $\Sigma_{\text {gas }}$ for 257 starforming regions, $520 \mathrm{pc}$ in diameter, in the disk of NGC 5194. They measured slopes of $N=1.37 \pm 0.03$ and $N=1.56 \pm 0.04$ for the molecular and total gas SFL, respectively. Bigiel et al. (2008) used far-UV and $24 \mu \mathrm{m}$ images to create a $\Sigma_{\mathrm{SFR}}$ map, and $21 \mathrm{~cm}, \mathrm{CO} J=2-1$, and CO $J=1-0$ data to create $\Sigma_{\text {gas }}$ maps of seven spiral galaxies and eleven late-type/dwarf galaxies. After convolving the maps to a common resolution of $750 \mathrm{pc}$, they performed a pixel-to-pixel analysis and measured a molecular SFL with an average $N=1.0 \pm 0.2$ for the normal spirals ( $N=0.84$ for NGC 5194). Both studies found a lack of correlation between the SFR and the atomic gas density, which saturates around a value of $10 M_{\odot} \mathrm{pc}^{-2}$. This value is thought to be associated with a density threshold for the formation of molecular gas, and is consistent with predictions from theoretical modeling of giant atomic-molecular complexes (Krumholz et al. 2009a). The total gas SFL is then driven by the correlation between the molecular gas density and the SFR, and the molecular fraction in the ISM. At the highest densities present in normal spiral galaxies $\left(\Sigma_{\mathrm{H}_{\mathrm{I}}+\mathrm{H}_{2}}=50-1000 \mathrm{M}_{\odot} \mathrm{pc}^{-2}\right)$ the ISM is mostly molecular and the total gas SFL closely follows the $\mathrm{H}_{2}$ SFL. At densities lower than $10 M_{\odot} \mathrm{pc}^{-2}$ the total gas SFL gets much steeper due to a strong decrease of the molecular fraction. This behavior has been recently modeled by Krumholz et al. (2009b).

While spatially resolved studies of the SFL obtain consistent results on the behavior of the atomic gas, they disagree when it comes down to the molecular component. The Bigiel et al. (2008) measurement of a linear molecular SFL is consistent with a scenario in which star formation occurs at a constant efficiency inside giant molecular clouds (GMCs), whose properties are fairly uniform across normal spiral galaxies (Blitz et al. 2007; Bolatto et al. 2008). This homogeneity in the properties of GMCs is expected if they are internally regulated by processes such as stellar feed-back, and they are decoupled from their surroundings due to the fact of being strongly overpressured (Krumholz et al. 2009b). Kennicutt et al. (2007), on the other hand, measured a super-linear molecular SFL in NGC 5194, which suggests an increasing star formation efficiency (SFE) toward higher gas densities. Although the authors state that a super-linear slope $(N>1)$ is still consistent with a constant "efficiency" if the star-forming lifetimes of massive clouds were systematically lower than those of low-mass clouds, this is true only if the efficiency is defined as the ratio of the produced stellar mass over the available molecular gas mass, which is the classical definition used by galactic studies in the Milky Way (MW). In this work, as well as in Bigiel et al. (2008), the efficiency is defined as $\mathrm{SFE}=\Sigma_{\mathrm{SFR}} / \Sigma_{\text {gas }}$, or the inverse of the depletion time, so shorter star formation timescales imply a higher SFE, and a super-linear SFL always translate in higher SFE at higher gas densities.

With the goal of investigating this issue, we have conducted the first measurement of the spatially resolved SFL using integral field spectroscopy. We mapped the $\mathrm{H} \alpha$ emission in the central $4.1 \times 4.1 \mathrm{kpc}^{2}$ of the nearby face-on spiral galaxy NGC 5194 using the Visible Integral field Replicable Unit Spectrograph Prototype (VIRUS-P; Hill et al. 2008). Hydrogen recombination lines are known to be good tracers of the SFR. Their intensity scales linearly with the ionizing UV flux in galaxies, which is dominated by the emission from massive stars $\left(\geqslant 10 M_{\odot}\right)$ with typical lifetimes of $<20 \mathrm{Myr}$; hence they provide an almost instantaneous measurement of the SFR (Kennicutt 1998a, and references therein).

Due to the small field of view of current integral field units (IFUs), typically less than $1 \operatorname{arcmin}^{2}$, two-dimensional spectroscopic H $\alpha$ mapping of nearby galaxies with large angular sizes has not been conducted efficiently in the past. Instead, narrow-band imaging has been typically used to construct $\mathrm{H} \alpha$-based SFR maps. H $\alpha$ narrow-band imaging suffers from contamination from the $\left[\mathrm{N}_{\mathrm{II}}\right] \lambda \lambda 6548,6584$ doublet, and is sensitive to systematic errors in continuum subtraction and the estimation of the strength of the $\mathrm{H} \alpha$ absorption in the underlying stellar spectrum. Spectroscopic measurements are free of all these sources of error, and hence provide a much cleaner measurement of $\mathrm{H} \alpha$ fluxes. A major part of this paper is dedicated to investigate these systematics in order to assess the validity of the typical corrections applied to narrow-band $\mathrm{H} \alpha$ images.

VIRUS-P is the largest field-of-view IFU in the world and it allows for efficient $\mathrm{H} \alpha$ mapping of nearby galaxies. The observations presented here were taken as part of the VIRUS-P Exploration of Nearby Galaxies (VENGA; ${ }^{1}$ G.A. Blanc et al. 2010 , in preparation). VENGA is a large-scale extragalactic IFU survey that will spectroscopically map large parts of the disks of $\sim 20$ nearby spirals, to allow a number of studies on star formation, structure assembly, stellar populations, gas and stellar dynamics, chemical evolution, ISM structure, and galactic feedback.

The VIRUS-P spectral map was used in combination with CO $J=1-0$ and H I $21 \mathrm{~cm}$ intensity maps of NGC 5194 from the Berkeley Illinois Maryland Array (BIMA) Survey of Nearby Galaxies (BIMA SONG; Helfer et al. 2003), and The H I Nearby Galaxy Survey (THINGS; Walter et al. 2008), to measure $\Sigma_{\mathrm{SFR}}, \Sigma_{\mathrm{H}_{2}}$, and $\Sigma_{\mathrm{H}_{\mathrm{I}}}$ in order to construct the spatially resolved SFL. In Sections 2 and 3, we present the VIRUS-P observations and the data reduction and calibration methods. In Section 4, we describe the $\mathrm{CO}$ and $21 \mathrm{~cm}$ data used to measure the molecular and atomic gas surface densities, as well as a HST NICMOS $\mathrm{Pa} \alpha$ image used to validate our dust extinction measurements. Section 5 presents the methods used to remove the stellar continuum and measure accurate nebular emission line fluxes, together with our dust extinction correction. The calculation of $\Sigma_{\text {gas }}$ is described in Section 6. The rejection of regions whose nebular emission is affected by the central active galactic nucleus (AGN) in NGC 5194 is presented in Section 7. The correction to account for the contribution of the diffuse ionized gas (DIG) to the $\mathrm{H} \alpha$ fluxes is described in Section 8. The resulting spatially resolved SFLs for the molecular, atomic, and total gas are presented in Section 9, followed by a discussion on the implications of our results for narrow-band imaging surveys in Section 10. Finally we compare our results with previous measurements and theoretical predictions of the SFL in Section 11, and present our conclusions in Section 12.

Throughout this paper we assume a distance to NGC 5194 of 8.2 Mpc for consistency with Kennicutt et al. (2007). While

\footnotetext{
http://www.as.utexas.edu/ gblancm/venga.html
} 

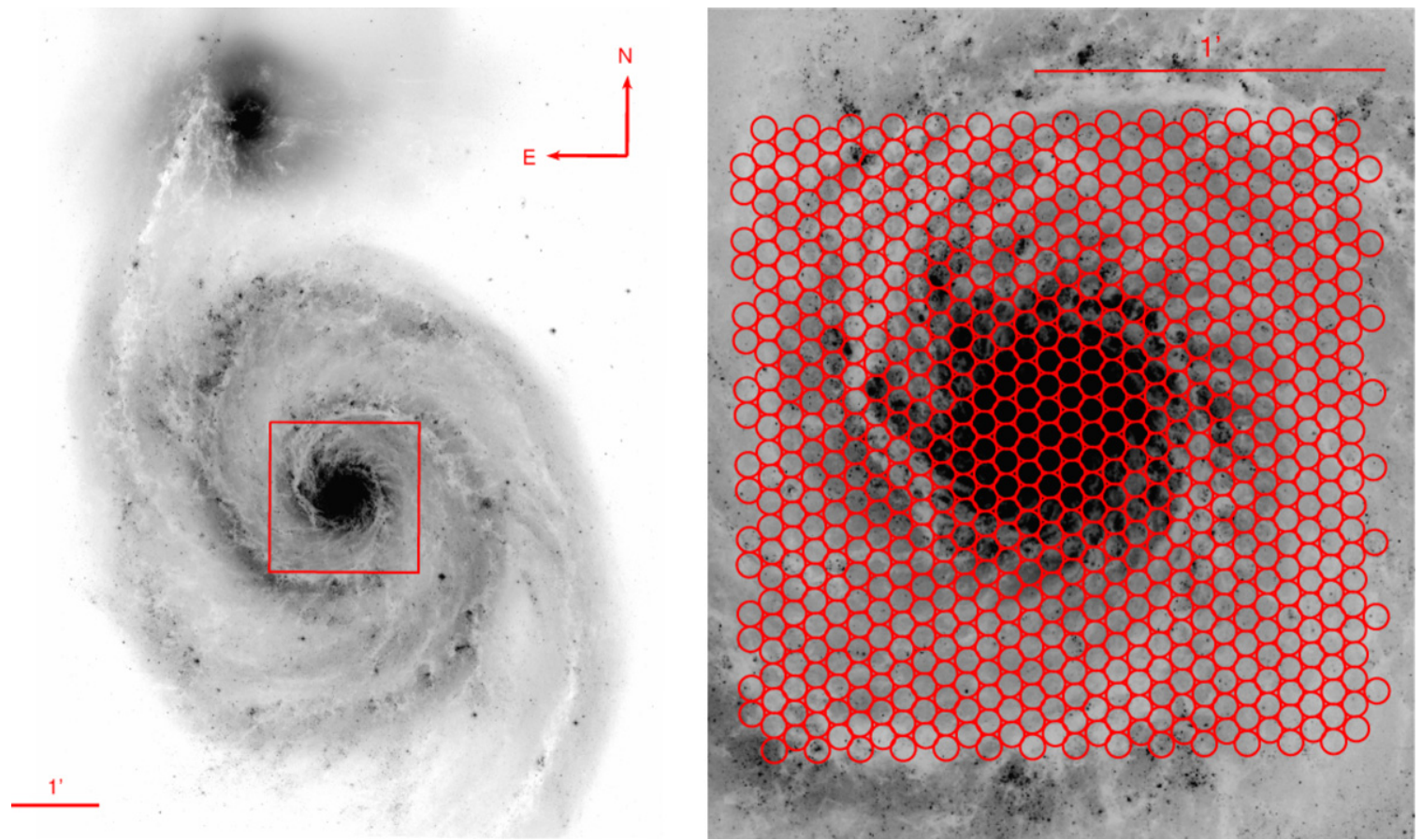

Figure 1. Left: $H S T+$ ACS $V$-band image of NGC5194 and its companion NGC 5195 (Mutchler et al. 2005). The central $4.1 \times 4.1 \mathrm{kpc}^{2}$ region sampled by the $1.7 \times 1.7$ VIRUS-P field of view is marked in red. Right: map of the 738 regions sampled by VIRUS-P in the three dither positions. Each region has a diameter of 4'. 3 corresponding to $\sim 170 \mathrm{pc}$ at the distance of NGC5194.

(A color version of this figure is available in the online journal.)

Bigiel et al. (2008) used a slightly smaller distance of $8.0 \mathrm{Mpc}$, it is worth noticing that most of the results in this paper are based on surface densities, which are independent of distance, and thus are not affected by the assumed value. All values for $\Sigma_{\mathrm{SFR}}$ are in units of $M_{\odot} \mathrm{yr}^{-1} \mathrm{kpc}^{-2}$, and values of $\Sigma_{\mathrm{gas}}$ are in units of $M_{\odot} \mathrm{pc}^{-2}$.

\section{OBSERVATIONS}

We obtained spatially resolved spectroscopy over the central $4.1 \times 4.1 \mathrm{kpc}^{2}$ region of NGC 5194 on the night of 2008 April 4, using VIRUS-P on the $2.7 \mathrm{~m}$ Harlan J. Smith telescope at McDonald Observatory. VIRUS-P with the VP-2 IFU bundle used in this work consists of a square array of 246 optical fibers, which samples a $1.7 \times 1.7$ field of view with a $1 / 3$ filling factor. The fibers are $200 \mu \mathrm{m}$ in diameter, corresponding to $4^{\prime \prime} .3$ on sky. The spectrograph images the spectrum of the 246 fibers on a $2048 \times 2048$ Fairchild Imaging CCD. Because of camera alignment issues, the spectrum of one fiber fell off the chip, reducing the number of usable fibers to 245 .

The spectrograph was used in a red setup under which it samples a wavelength range of 4570-6820 $\AA$ with a spectral resolution of $\sim 5.0 \AA$ (FWHM). This red setup allows us to sample both $\mathrm{H} \beta$ and $\mathrm{H} \alpha$, and our resolution is high enough to resolve the $\mathrm{H} \alpha-[\mathrm{N}$ II $] \lambda \lambda 6548,6893$ complex. We took the data in $2 \times 1$ binning mode in the spectral direction which translates into a plate scale of $2.2 \AA$ pixel $^{-1}$. Given the $1 / 3$ filling factor of the IFU, three dithered exposures were necessary to sample the complete field of view.

We obtained four 20 minute exposures at each of the three dither positions, accounting for an effective exposure time of 80 minutes. Dither 1 was centered at $\alpha=13: 29: 52.69 ; \delta=+47: 11: 43.0$. Dithers 2 and 3 were offset from dither 1 by $\Delta \alpha=-3^{\prime \prime} .6 ; \Delta \delta=-2^{\prime \prime} .0$ and $\Delta \alpha=0^{\prime \prime} 0 ; \Delta \delta=$
-4 .'0, respectively. Figure 1 shows the observed region in NGC 5194 as well as the position of the IFU fibers for the three dithers. Because of the extended nature of NGC 5194 no fibers in the field of view sampled a blank region of the sky. This implied the need for off-source sky frames in between science frames. We obtained 5 minute sky exposures bracketing all science exposures. These were obtained $30^{\prime}$ north of NGC 5194. The typical seeing during the observations was 2 .'0.

Bias frames, comparison $\mathrm{NeCd}$ lamps, and twilight flats were taken at the beginning and end of the night. VIRUS-P is mounted on a two degree of freedom gimble at the broken Cassegrain focus of the telescope. The gimble keeps the spectrograph in a fixed gravity vector independent of the position of the telescope during the observations which translates into a practically complete lack of flexure in the spectrograph optical components. For this reason calibration frames intercalated with the science observations were not necessary.

The spectro-photometric standard Feige 34 was observed for the purpose of flux calibration (see Section 3.1). Standard observations were performed using a finer 6 position dither pattern which better samples the point-spread function (PSF) of the star and ensures the collection of its total flux (see Section 3.1 and Figure 2).

The instrument is equipped with a guiding camera which images a $4.5 \times 4.5$ field offset from the science field sampled by the IFU. The guiding camera is a $512 \times 512$ pixel Apogee unit equipped with a BV filter which allows broadband photometric measurements of the stars in the field. During the night we saved a guider frame every $30 \mathrm{~s}$ in order to reconstruct changes in atmospheric transparency. The guider images are also used to establish the IFU astrometry. The relative offset, rotation, and plate scales of the guider and IFU fields have been precisely calibrated using observations of crowded fields in open clusters, so the pixel coordinates of stars in the guider frames provide us 

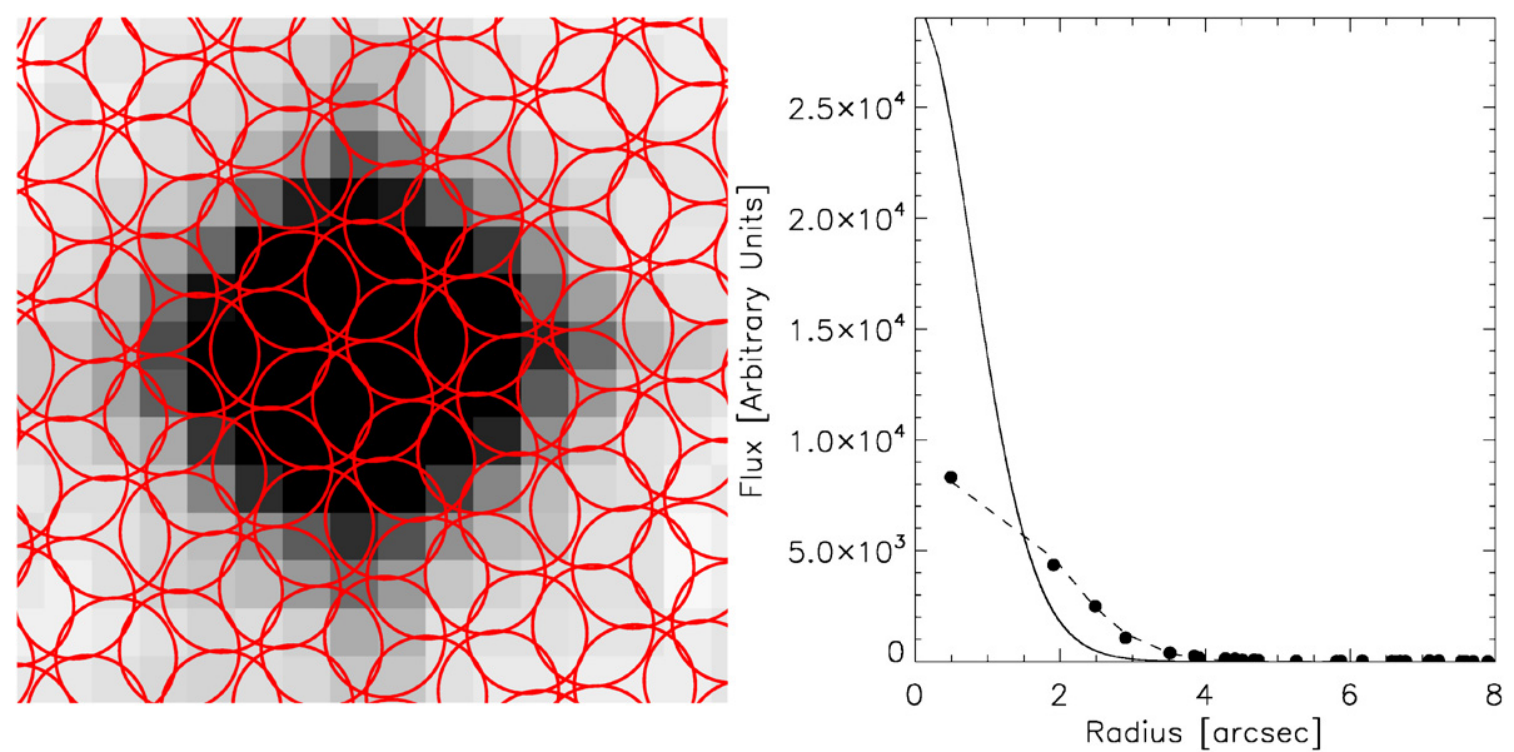

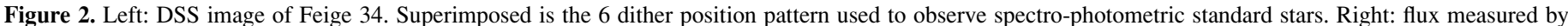

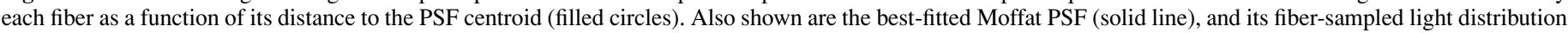
(dashed line).

(A color version of this figure is available in the online journal.)

with coordinates for the center of all fibers in the IFU with an astrometric rms of $\sim 0^{\prime \prime} .5$.

In this way we obtained spectra for 735 regions 4.3 in diameter $(\sim 170 \mathrm{pc}$ at the distance of NGC 5194$)$, in the central region of the galaxy. The spectra reaches a median $5 \sigma$ sensitivity in continuum flux density of $2.5 \times 10^{-17} \mathrm{erg} \mathrm{s}^{-1} \mathrm{~cm}^{-2} \AA^{-1}$, which translates into a median signal-to-noise ratio $(\mathrm{S} / \mathrm{N})$ per resolution element of 95 (53 for the faintest fiber).

\section{DATA REDUCTION}

Data reduction is performed using our custom pipeline VACCINE (J. Adams et al. 2010, in preparation). Individual frames are overscan and bias subtracted, and bad pixels are masked. We use the twilight flats to trace the peak of the spatial profile of the spectrum of all fibers on the chip, and extract the two-dimensional spectrum of each fiber on the science frames, comparison lamp frames, and flats using a 7 pixel aperture around the peak.

The extracted comparison lamp spectra are used to compute an independent wavelength solution for each fiber. We use 4th order polynomials to compute the wavelength solutions which show a typical rms of $0.2 \AA$ ( $~ 0.1$ pixel).

We correct the twilight flats for solar absorption lines and use them to measure the shape and amplitude of the spatial profile of the fibers as a function of wavelength. This profile is given by the PSF of the fibers on chip in the spatial direction, and the relative instrumental throughput of each fiber as a function of wavelength. Dividing the twilight flats by this profile yields a pixel-to-pixel flat. We divide all science, sky, and spectrophotometric standard frames by both the fiber profile and the pixel-to-pixel flats. This removes any fiber-to-fiber and pixelto-pixel variations in sensitivity.

A background frame is created for each science exposure by averaging the two bracketing 5 minute sky frames and scaling by the difference in exposure time. We estimate the sky spectrum for each fiber by fitting a non-uniform spline to the spectra of the 60 neighboring fibers on chip in the background frame. This spectrum is subtracted from each fiber in the science data.
In order to test the quality of our background subtraction algorithm we construct background frames for each of our sky exposures using the two closest of the other sky exposures. We then follow the same procedure to background subtract our sky frames. We observe residuals centered around zero in the background subtracted sky frames that are less than $1 \%$ of the galaxy continuum flux in the faintest fibers in our science data. The only exception are the regions of the spectra at the wavelength of the four brightest sky emission lines in our wavelength range in which the residuals can be considerably larger due to the fast time variability of these spectral features. These regions showing poor background subtraction are masked in our science data. At this stage we combine individual exposures using a biweight (Beers et al. 1990).

Error maps including Poisson photon count uncertainties and read-noise are created for every fiber on each frame. We use these error maps together with the fiber profile to calculate the weights used for collapsing the two-dimensional spectrum into a one-dimensional spectrum. The flux in photoelectrons at each wavelength after collapsing is given by

$$
f_{\lambda}=\frac{\sum_{i=1}^{7}\left(\frac{p_{i}}{e_{i}}\right)^{2} G f_{\lambda, i}}{\sum_{i=1}^{7}\left(\frac{p_{i}}{e_{i}}\right)^{2}},
$$

where $p_{i}$ is the value of the fiber profile, $G$ is the gain, $f_{i}$ is the flux in ADUs in the combined background-subtracted spectrum, and $e_{i}$ is the corresponding error at each pixel as measured in the error map. This is equivalent to weighting the pixels by $(\mathrm{S} / \mathrm{N})^{2}$. The sum is performed at every wavelength (column) over the 7 pixel aperture used for extraction. The final product is a wavelength-calibrated one-dimensional spectrum of the area sampled by each of the 245 fibers on each of the three dither positions on the galaxy.

\subsection{Flux Calibration}

Flux calibration of IFU data can be challenging but, if proper care is taken, very accurate spectro-photometry can be achieved. 
This is mostly because of the lack of a wavelength-dependent slit loss function. Atmospheric dispersion can change the position of a standard star in the field of view as a function of wavelength, but as long as the field is completely sampled by the fibers the total flux of the star at all wavelengths is always collected. Also, photometry of stars in the guider images taken during the observations allows us to measure and correct for changes in atmospheric transparency during the night.

During the observation of standard stars, fibers in the IFU only sample a region of the star's PSF. Determining the fraction of the total flux collected by each fiber is essential in order to compute a proper instrumental sensitivity function by comparing each fiber spectrum to the total intrinsic spectrum of the star. This requires knowledge of the shape of the PSF as well as the distance from the PSF centroid to the center of each fiber.

The spectro-photometric standard star Feige 34 was observed using a 6 position dither pattern shown in the left panel of Figure 2. This tight pattern provides a better sampling of the PSF and ensures we collect the total flux of the star. We calculate the position of the centroid of the PSF relative to the fibers by taking the weighted average of the fibers positions in the field of view, using the measured flux in each of them as weights. This corresponds to the first moment of the observed light distribution.

The filled circles in the right panel of Figure 2 show the flux measured in each fiber as a function of its radial distance to the PSF center. This information can be used to reconstruct the shape of the star PSF at the moment of the observations. In order to do this, we assume a Moffat profile for the PSF and reconstruct its observed light distribution by summing the flux in 4".3 diameter circular apertures at the corresponding radial distance of each fiber. The best-fitted PSF and its fiber-sampled light distribution are shown by the solid and dashed curves in the right panel of Figure 2. It can be seen that the best-fitted model PSF, after being sampled by the fibers in our dither pattern, matches the measured flux remarkably. This PSF model allows us to know what fraction of the star total flux was measured by each fiber during the observations.

We normalize the spectrum of each fiber by the fraction of the total flux it sampled, and average this value for all fibers having a significant $(>5 \sigma)$ flux measurement in order to obtain the star total instrumental spectrum. We correct the total spectrum by atmospheric extinction and use the Feige 34 measurement of Oke (1990) to construct our instrumental sensitivity function.

Relative variations in atmospheric transparency during the night are measured by performing aperture photometry on stars in the guider images. Observing conditions were confirmed to be very stable, with maximum variations in transparency of less than a $10 \%$. All spectra in our science frames are corrected by these variations, atmospheric extinction, and flux calibrated using the instrumental sensitivity function.

It is important to note that any difference in illumination or throughput between fibers was taken out during the flat-fielding process, so a common sensitivity function applies to all fibers. Our final product is a wavelength and flux-calibrated spectrum for the 735 regions.

In order to estimate the systematic uncertainty in our flux calibration we have compared sensitivity functions computed using different standard star observations taken as part of different observing programs with VIRUS-P. Comparison of 10 standards taken between September 2007 and June 2008 under different observing conditions show that after correcting for relative changes in atmospheric transparency (using photometry of stars in the guider images) the computed sensitivity functions show an rms scatter of less than $5 \%$.

\section{OTHER DATA}

\subsection{THINGS H I Data}

We use a combined $21 \mathrm{~cm}$ line intensity map of NGC 5194 from the Very Large Array (VLA) ${ }^{2}$ taken as part of THINGS ${ }^{3}$ (Walter et al. 2008) to estimate the atomic gas surface density $\left(\Sigma_{\mathrm{H}_{\mathrm{I}}}\right)$. H I data for NGC 5194 were taken using the B, C and D arrays during 2004 and 2005, with a combined on source integration time of $\sim 10 \mathrm{hr}$. The final co-added ( $\mathrm{B}+\mathrm{C}+\mathrm{D}$ array) integrated intensity map has a robustly weighted beam size of $5^{\prime \prime} .82 \times 55^{\prime \prime} .56$, which is well matched to the 4".3 VIRUS-P fiber diameter convolved with the $2^{\prime \prime}$ seeing. The $1 \sigma$ noise per $5.2 \mathrm{~km} \mathrm{~s}^{-1}$ channel is $0.44 \mathrm{mJy}$ beam $^{-1}$ corresponding to a atomic gas surface density of $\Sigma_{\mathrm{HI}}=0.59 M_{\odot} \mathrm{pc}^{-2}$. For more details on data products and data reduction see Walter et al. (2008).

\subsection{BIMA SONG CO Data}

Molecular gas surface densities are measured using the $\mathrm{CO}$ $J=1-0$ intensity map of NGC 5194 from the BIMA SONG (Helfer et al. 2003). Zero-spacing single dish data from the NRAO 12 m telescope were combined with the interferometric BIMA $\mathrm{C}$ and $\mathrm{D}$ array data, resulting in a map with a robust beam size of $5^{\prime \prime} .8 \times 5^{\prime \prime} .1$, well matched to the $21 \mathrm{~cm}$ map and the VIRUS-P spatial resolution. The corresponding $1 \sigma$ noise is $61 \mathrm{mJy}$ beam $^{-1}$ in a $10 \mathrm{~km} \mathrm{~s}^{-1}$ channel or $\Sigma_{\mathrm{H}_{2}}=13 M_{\odot} \mathrm{pc}^{-2}$. For more details on the observations and the data reduction refer to Helfer et al. (2003).

\subsection{HST NICMOS Paschen- $\alpha$ Data}

The center of NGC 5194 was imaged in Pa $\alpha$ by Scoville et al. (2001) using $H S T+$ NICMOS. A $3 \times 3$ mosaic covering the central $186^{\prime \prime} \times 188^{\prime \prime}$ of the galaxy was imaged using the F187N and F190N narrow-band filters, sampling the $\mathrm{Pa} \alpha$ line and the neighboring stellar continuum, respectively. In this work, we use this continuum-subtracted $\mathrm{Pa} \alpha$ image to measure emission line fluxes to check the validity of our dust extinction correction. The data reduction, mosaicking, flux calibration, and continuum subtraction are described in Scoville et al. (2001) and Calzetti et al. (2005). The $\mathrm{Pa} \alpha$ image overlaps completely with the VIRUS-P pointing shown in Figure 1.

\section{MEASUREMENT OF EMISSION LINE FLUXES}

We estimate the current $\Sigma_{\mathrm{SFR}}$ for each region by means of the $\mathrm{H} \alpha$ nebular emission luminosity. In this section, we describe the methods used to separate the emission lines coming from ionized gas from the underlying stellar spectrum, measure emission line fluxes, and estimate the dust extinction in each region using the $\mathrm{H} \alpha / \mathrm{H} \beta$ ratio.

\footnotetext{
2 The VLA is operated by the National Radio Astronomy Observatory, which is a facility of the National Science Foundation, operated under cooperative agreement by Associated Universities, Inc.

3 http://www.mpia.de/THINGS/Data.html

4 http://nedwww.ipac.caltech.edu/level5/March02/SONG/SONG.html
} 

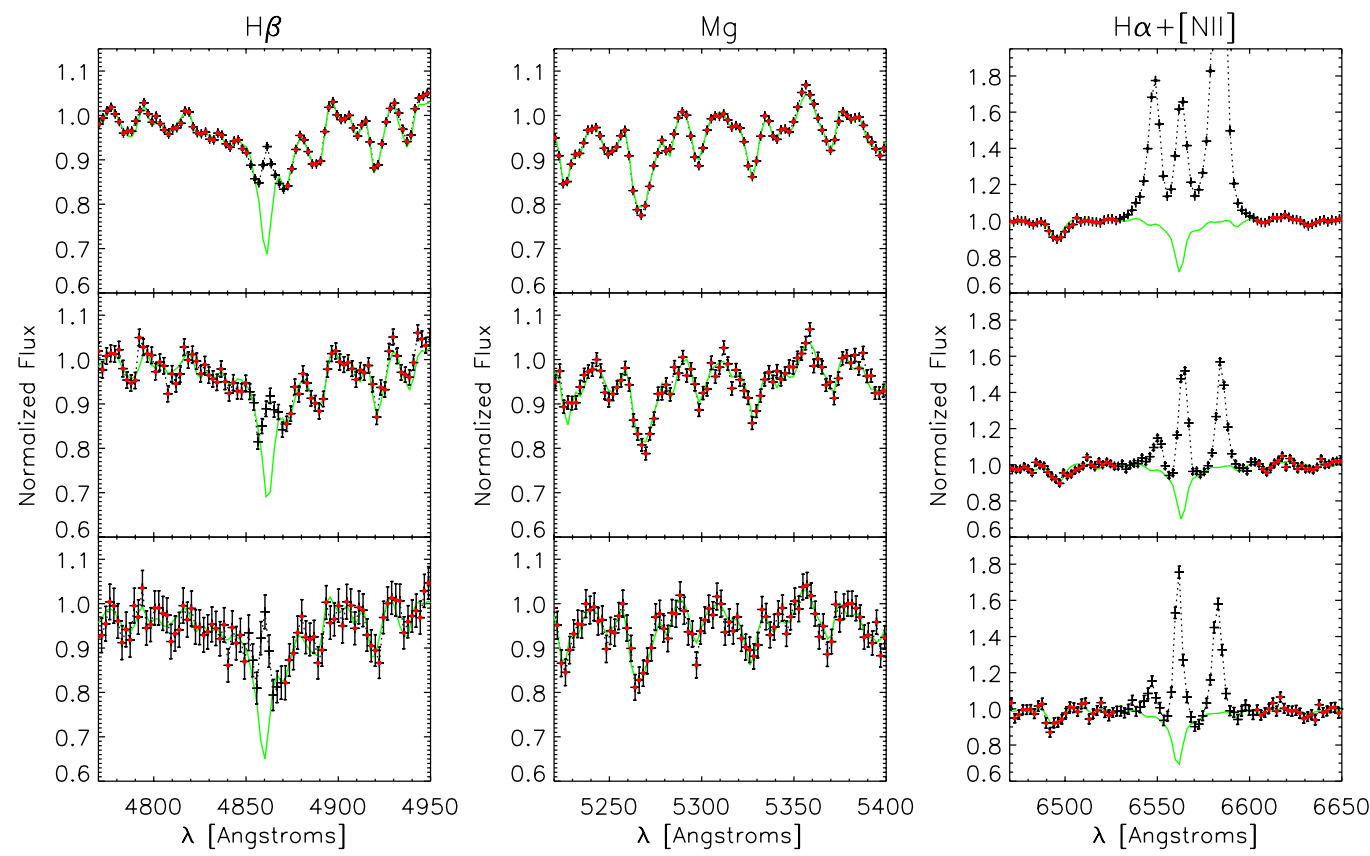

Figure 3. Continuum normalized spectra around the $\mathrm{H} \beta, \mathrm{Mg}$ II, and $\mathrm{H} \alpha$ features for three regions having the highest, median, and lowest (top, middle, bottom, respectively) S/N per resolution element in the continuum. Crosses show the data with error bars. Red crosses mark the data points used to fit the best linear combination of stellar templates (green solid line). Black crosses were masked in the fit due to the presence of nebular emission.

(A color version of this figure is available in the online journal.)

\subsection{Photospheric Absorption Lines and Continuum Subtraction}

In galaxy spectra, both the $\mathrm{H} \alpha$ and $\mathrm{H} \beta$ emission lines sit on top of strong Balmer absorption lines characteristic of the photospheric stellar spectrum of young stars. Removing the contribution from these absorption lines is essential in order to estimate properly the emission line flux.

We use a linear combination of stellar template spectra to fit the absorption line spectrum of each region. The templates are high-S/N, high-resolution, continuum normalized spectra of a set of 18 stars from the Indo-U.S. Library of Coudé Feed Stellar Spectra (Valdes et al. 2004). The stars were chosen to span a wide range in spectral types and metallicities (A7 to K0, and $[\mathrm{Fe} / \mathrm{H}]$ from -1.9 to 1.6$)$.

The resolution of the templates is degraded to match the VIRUS-P 5.0 A spectral resolution. For each of the 735 regions, we mask the parts of the galaxy spectrum affected by emission lines and sky subtraction residuals from bright sky lines. The continuum at each wavelength is estimated using an iterative running median filter, and used to normalize the observed spectrum.

We use this masked, continuum normalized spectrum to fit the best linear combination of stellar templates for each region. Figure 3 shows the best-fitted template combinations in regions centered in $\mathrm{H} \beta, \mathrm{Mg}$ b and $\mathrm{H} \alpha$ for three regions in the galaxy. The bottom, middle, and upper panels correspond to fibers with the lowest, median, and highest $\mathrm{S} / \mathrm{N}$ in their spectra, respectively. For all 735 regions we obtain excellent fits to the underlying stellar spectrum. Figure 3 shows the importance of taking into account the effect of photospheric Balmer absorption lines when measuring $\mathrm{H} \alpha$ and $\mathrm{H} \beta$ fluxes. Ignoring the presence of the absorption features can introduce serious underestimations of the emission line fluxes. For $\mathrm{H} \alpha$ this effect can account for underestimations of up to $100 \%$ as will be shown in Section 10 .

The best-fitted linear combination of stellar templates is scaled by the galaxy continuum and subtracted from the original spectrum in order to produce pure nebular emission line spectra for all fibers. Figure 4 shows the nebular spectrum of the same regions shown in Figure 3. After subtracting the stellar light, we are able to identify most well-known emission features in galaxy spectra. $\mathrm{H} \beta$, [O III] $\lambda \lambda 4959,5007$, [N II] $\lambda \lambda, 6548,6584$, $\mathrm{H} \alpha$ and $[\mathrm{S} \mathrm{II}] \lambda \lambda, 6717,6731$ are clearly seen in the spectra of all 735 regions. Visual inspection of Figure 4 shows that the $[\mathrm{N}$ II $] \lambda \lambda, 6548,6584 / \mathrm{H} \alpha$ ratio can change drastically from region to region. This effect can introduce systematic biases in narrowband-measured $\mathrm{H} \alpha$ fluxes if the ratio is assumed to be constant across the disk (Calzetti et al. 2005; Kennicutt et al. 2007). This issue will be discussed in detail in Section 10.

\subsection{Emission Line Fluxes}

We measure emission line fluxes by independently fitting $\mathrm{H} \beta$, [O III] $] \lambda 5007$, the $\mathrm{H} \alpha-\left[\mathrm{N}_{\mathrm{II}}\right] \lambda \lambda, 6548,6584$ complex, and the [S II $] \lambda \lambda, 6717,6731$ doublet. Although the lines in the $\mathrm{H} \alpha$-[N II] complex are clearly resolved in our spectra, their wings show some level of overlap so we used a 3 Gaussian component model to fit these lines. Similarly a 2 Gaussian component model was used to fit the [S $\left.\mathrm{SI}_{\mathrm{II}}\right]$ doublet. $\mathrm{H} \beta$ and $\left[\mathrm{O}_{\mathrm{III}}\right] \lambda 5007$ were fitted using single Gaussians. These fits provide the total flux and its uncertainty of all the above lines for the 735 regions. All lines are detected with a significance higher than $3 \sigma$ in all fibers. We measure a median and the lowest $\mathrm{S} / \mathrm{N}$ over all fibers of 109 and 15 for $\mathrm{H} \alpha, 29$ and 4 for $\mathrm{H} \beta, 49$ and 13 for [N $\mathrm{II}] \lambda 6584$, and 32 and 5 for $[\mathrm{S}$ II] $] \lambda 6717$. Emission line fluxes of all lines for all fibers are given in Table 1, available in the electronic version of this paper.

\subsection{Extinction Correction from the Balmer Decrement}

The observed spectra are affected by differential extinction due to the presence of dust in the ISM of both NGC 5194 and the MW. Before attempting to estimate SFRs from $\mathrm{H} \alpha$ fluxes, these have to be corrected for dust extinction. Failing to do so can introduce underestimations in the SFR of up to factors of $\sim 10$ 


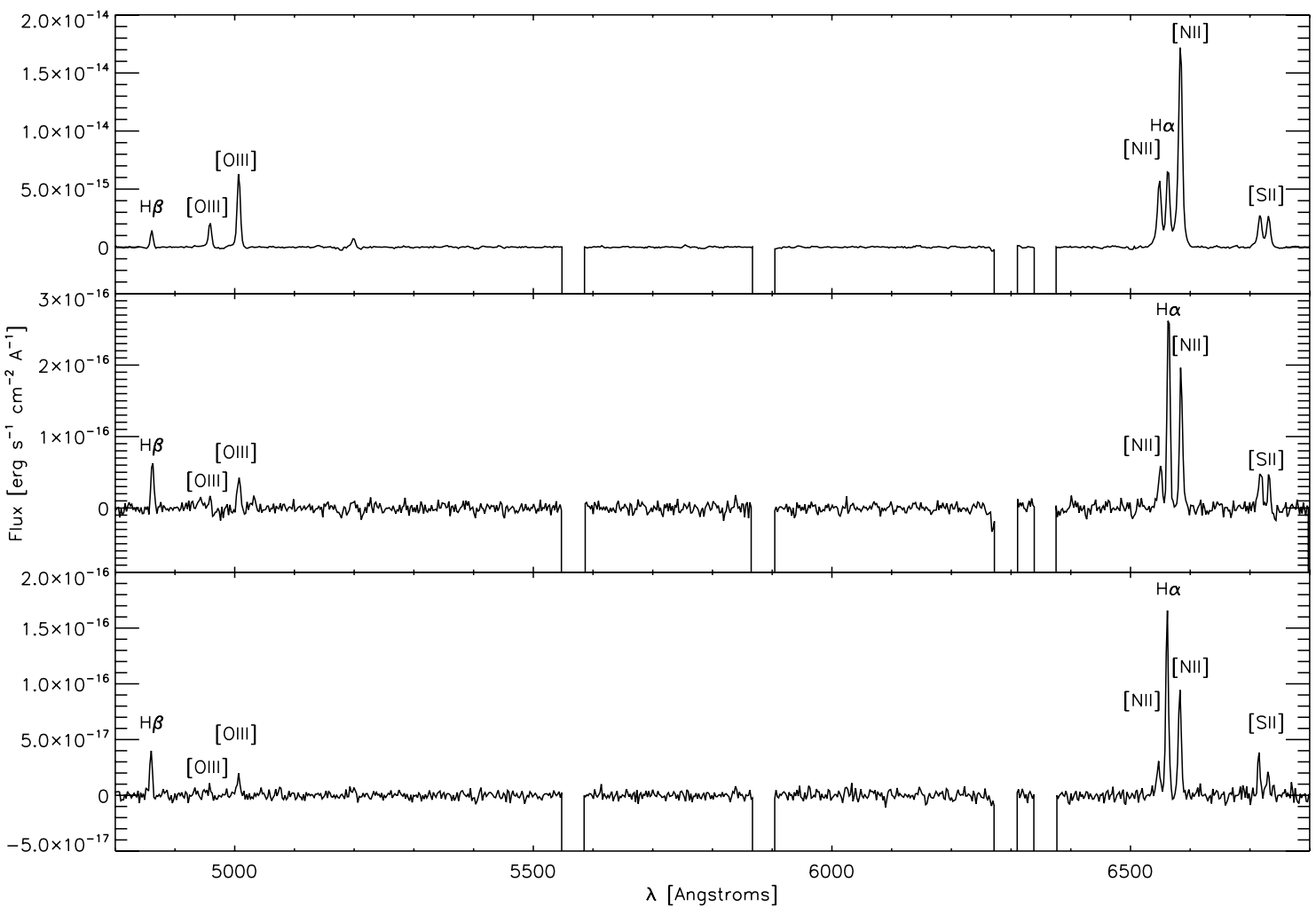

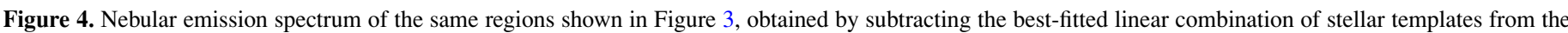
observed spectrum. Masked parts of the spectra correspond to the regions around strong night sky emission lines showing background subtraction residuals.

in the regions we are studying. The Balmer line ratio $\mathrm{H} \alpha / \mathrm{H} \beta$, as will be shown bellow, provides a good estimate of the dust extinction at the wavelength of the $\mathrm{H} \alpha$ line.

Assuming an intrinsic $\mathrm{H} \alpha / \mathrm{H} \beta$ ratio of 2.87 (Osterbrock \& Ferland 2006), the observed ratio provides the extinction at the wavelength of $\mathrm{H} \alpha$ through the following equation,

$$
A_{\mathrm{H} \alpha}=-2.5 \log \left[\frac{[\mathrm{H} \alpha / \mathrm{H} \beta]_{\mathrm{obs}}}{2.87}\right]\left(\frac{1}{1-k(\mathrm{H} \alpha) / k(\mathrm{H} \beta)}\right) \text {, }
$$

where $[\mathrm{H} \alpha / \mathrm{H} \beta]_{\mathrm{obs}}$ is the observed line ratio and $k(\lambda)$ is the extinction law. We assume a foreground MW extinction law as parameterized by Pei (1992). SMC and LMC laws were also tested (also using the Pei 1992 parametrization), and no significant change was observed in the deduced extinction values (these three extinction laws are practically identical at these wavelengths). To correct for Galactic extinction toward NGC 5194 we use a value of $A_{B}=0.152$, taken from Schlegel et al. (1998).

In order to test the reliability of our Balmer decrement extinction values, we compare our corrected $\mathrm{H} \alpha$ fluxes with the corrected $\mathrm{Pa} \alpha$ fluxes. The hydrogen recombination $\mathrm{Pa} \alpha$ line at $1.87 \mu \mathrm{m}$, although one order of magnitude fainter than $\mathrm{H} \alpha$, is very weakly absorbed by dust, and hence provides an unbiased estimate of the intrinsic SFR even in highly extincted regions (Scoville et al. 2001). Most recent studies of spatially resolved star formation in nearby disk galaxies use recipes to account for dust obscured star formation which are ultimately linked to a calibration based on $\mathrm{Pa} \alpha$ (Calzetti et al. 2005; Kennicutt et al. 2007; Bigiel et al. 2008; Leroy et al. 2008). In particular, Calzetti et al. (2005) find a tight linear correlation between the $24 \mu \mathrm{m}$ luminosity of star-forming regions in NGC 5194 and their $\mathrm{P} \alpha$ luminosities, providing justification for the use of linear combinations of $24 \mu \mathrm{m}$ fluxes with either $\mathrm{H} \alpha$ or UV fluxes to estimate the intrinsic SFR in the other mentioned works. In our case, if the extinction-corrected $\mathrm{H} \alpha$ fluxes linearly correlate with the corrected $\mathrm{Pa} \alpha$ fluxes, following the intrinsic line ratio expected from recombination theory, then we can confirm that our extinction values have been properly estimated. In that case we can do without the IR data, and apply an extinction correction to the measured $\mathrm{H} \alpha$ fluxes which is solely based on the optical spectra.

We measure $\mathrm{P} \alpha$ fluxes for all 735 regions in the NICMOS F187N continuum-subtracted narrow-band image (see Section 4.1), using apertures matching the size of the VIRUS-P fibers. Figure 5 shows extinction-corrected $\mathrm{Pa} \alpha$ versus $\mathrm{H} \alpha$ fluxes for all regions showing $5 \sigma$ detections of $\mathrm{Pa} \alpha$ emission in the NICMOS narrow-band image. Both lines have been corrected using the Balmer decrement derived extinction, and a MW extinction law. The solid line in Figure 5 shows the theoretical $\mathrm{H} \alpha / \mathrm{Pa} \alpha=8.15$ ratio taken from Osterbrock \& Ferland (2006). The observed line ratios are in agreement with the theoretical value, and the scatter can be attributed mostly to measurement errors. This confirms that $\mathrm{H} \alpha$ fluxes, once corrected for dust obscuration using the Balmer decrement derived extinction, can provide an unbiased measure of the intrinsic SFR in the disks of normal face-on spirals.

\section{MEASUREMENT OF GAS MASS SURFACE DENSITIES}

In order to measure the atomic and molecular gas surface density at the position of each of the 735 regions under study, we measure integrated intensities in the THINGS $21 \mathrm{~cm}$ and the BIMA SONG CO $J=1-0$ maps, and translate them into gas surface densities using the calibrations presented below. The intensities are measured over an area equal to the beam size of each map. At each of the 735 fiber positions we perform aperture photometry on the $21 \mathrm{~cm}$ and $\mathrm{CO}$ maps, and measure the integrated gas intensity in apertures of effective radius 
Table 1

Nebular Emission Line Fluxes, Gas Surface Densities, and SFR Surface Densities

\begin{tabular}{|c|c|c|c|c|c|c|c|c|c|c|}
\hline ID & Equatorial Coordinates & $\mathrm{H} \beta$ & {$\left[\mathrm{N}_{\text {II }}\right] \lambda 6548$} & $\mathrm{H} \alpha$ & {$\left[\mathrm{N}_{\text {II }}\right] \lambda 6584$} & {$\left[\mathrm{~S}_{\mathrm{II}}\right] \lambda 6717$} & {$\left[\mathrm{~S}_{\text {II }}\right] \lambda 6731$} & $\overline{\Sigma_{\mathrm{H} 2}}$ & $\overline{\Sigma_{\mathrm{HI}_{\mathrm{I}}}}$ & $\overline{\Sigma_{\mathrm{SFR}}}$ \\
\hline & $\alpha$ & & & $\left(10^{-16} \mathrm{erg} \mathrm{s}^{-1} \mathrm{~cm}^{-2}\right)$ & & & & $\left(M_{\odot} \mathrm{pc}^{-2}\right)$ & $\left(M_{\odot} \mathrm{pc}^{-2}\right)$ & $\left(M_{\odot} \mathrm{yr}^{-1} \mathrm{kpc}^{-2}\right)$ \\
\hline 1 & $13: 29: 48.11+47: 12: 35.6$ & $12.57 \pm 0.11$ & $6.45 \pm 0.42$ & $69.73 \pm 0.59$ & $17.63 \pm 0.43$ & $6.89 \pm 0.23$ & $4.95 \pm 0.23$ & $10.71 \pm 13.00$ & $9.75 \pm 0.59$ & $0.08181 \pm 0.01652$ \\
\hline 2 & $13: 29: 48.83+47: 12: 35.5$ & $4.22 \pm 0.41$ & $4.94 \pm 0.46$ & $29.44 \pm 0.54$ & $13.90 \pm 0.48$ & $4.77 \pm 0.43$ & $3.12 \pm 0.43$ & $127.44 \pm 13.00$ & $12.25 \pm 0.59$ & $0.05385 \pm 0.01664$ \\
\hline 3 & $13: 29: 49.55+47: 12: 35.3$ & $6.05 \pm 0.25$ & $5.89 \pm 0.43$ & $36.35 \pm 0.52$ & $17.82 \pm 0.47$ & $6.02 \pm 0.10$ & $4.63 \pm 0.10$ & $399.25 \pm 13.00$ & $8.73 \pm 0.59$ & $0.04681 \pm 0.01063$ \\
\hline 4 & $13: 29: 50.28+47: 12: 35.2$ & $4.78 \pm 0.25$ & $4.42 \pm 0.53$ & $27.71 \pm 0.59$ & $13.85 \pm 0.55$ & $4.11 \pm 0.10$ & $3.02 \pm 0.10$ & $174.20 \pm 13.00$ & $10.05 \pm 0.59$ & $0.02975 \pm 0.00752$ \\
\hline 5 & $13: 29: 51.00+47: 12: 35.0$ & $2.94 \pm 0.01$ & $4.88 \pm 0.55$ & $16.37 \pm 0.54$ & $11.66 \pm 0.54$ & $3.19 \pm 0.03$ & $2.44 \pm 0.03$ & $127.30 \pm 13.00$ & $10.53 \pm 0.59$ & $0.01123 \pm 0.00274$ \\
\hline 6 & $13: 29: 51.72+47: 12: 34.8$ & $4.75 \pm 0.04$ & $5.77 \pm 0.29$ & $24.74 \pm 0.31$ & $16.43 \pm 0.30$ & $4.64 \pm 0.10$ & $2.99 \pm 0.10$ & $131.55 \pm 13.00$ & $7.08 \pm 0.59$ & $0.01791 \pm 0.00369$ \\
\hline 7 & $13: 29: 52.44+47: 12: 34.7$ & $10.97 \pm 0.54$ & $7.33 \pm 0.27$ & $54.63 \pm 0.36$ & $21.14 \pm 0.30$ & $6.39 \pm 0.26$ & $4.75 \pm 0.25$ & $177.73 \pm 13.00$ & $8.80 \pm 0.59$ & $0.04645 \pm 0.01083$ \\
\hline 8 & $13: 29: 53.16+47: 12: 34.5$ & $4.62 \pm 0.48$ & $4.20 \pm 0.44$ & $27.62 \pm 0.50$ & $13.26 \pm 0.47$ & $4.30 \pm 0.48$ & $2.47 \pm 0.45$ & $168.82 \pm 13.00$ & $8.98 \pm 0.59$ & $0.03255 \pm 0.01115$ \\
\hline 9 & $13: 29: 53.89+47: 12: 34.3$ & $4.99 \pm 0.44$ & $4.08 \pm 0.25$ & $23.63 \pm 0.28$ & $11.96 \pm 0.27$ & $4.13 \pm 0.47$ & $2.97 \pm 0.48$ & $234.42 \pm 13.00$ & $7.61 \pm 0.59$ & $0.01148 \pm 0.00453$ \\
\hline 10 & $13: 29: 54.61+47: 12: 34.2$ & $14.64 \pm 0.58$ & $7.49 \pm 0.53$ & $77.49 \pm 0.77$ & $23.63 \pm 0.59$ & $8.51 \pm 0.27$ & $6.00 \pm 0.27$ & $156.60 \pm 13.00$ & $10.39 \pm 0.59$ & $0.08200 \pm 0.01802$ \\
\hline
\end{tabular}

(This table is available in its entirety in a machine-readable form in the online journal. A portion is shown here for guidance regarding its form and content.) 


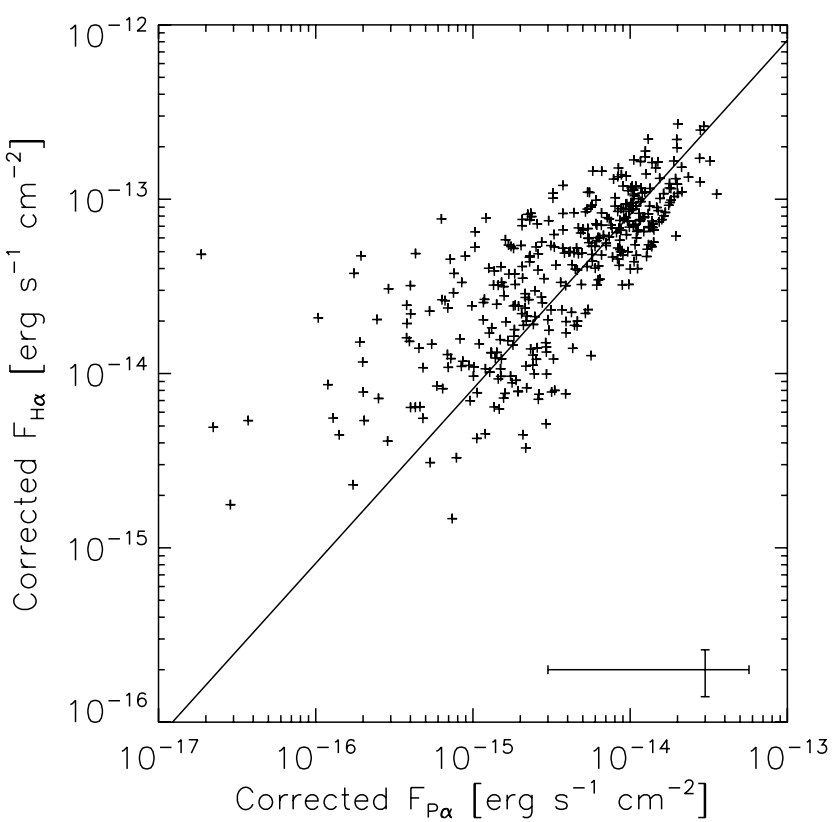

Figure 5. $\mathrm{H} \alpha$ vs. $\mathrm{Pa} \alpha$ fluxes of all regions showing $5 \sigma$ detections of $\mathrm{Pa} \alpha$ emission in the NICMOS narrow-band image. Fluxes are corrected for dust extinction using the Balmer decrement derived values. The solid line shows the $\mathrm{H} \alpha / \mathrm{Pa} \alpha=8.15$ ratio predicted by recombination theory. Median error bars for the corrected fluxes are shown.

$r_{\text {eff }}=\sqrt{a b} / 2$, where $a$ and $b$ are the major and minor axes of the beam of each map. This translates in an effective aperture diameter of 5.7 and 5".4 for the $21 \mathrm{~cm}$ and CO maps respectively, which is well matched with the VIRUS-P spatial resolution which is set by the convolution of a 4". 3 diameter fiber and a $2^{\prime \prime}$ FWHM seeing disk.

To convert the $21 \mathrm{~cm}$ intensities in atomic hydrogen column densities we use the following relation adapted from Walter et al. (2008),

$$
N_{\mathrm{HI}}=1.823 \times 10^{18}\left(\frac{T_{B}}{\mathrm{~K} \mathrm{~km} \mathrm{~s}^{-1} \mathrm{sr}}\right) \mathrm{cm}^{-2},
$$

where $T_{B}$ is the velocity integrated surface brightness temperature in the $21 \mathrm{~cm}$ map. To convert the CO $J=1-0$ intensities to $\mathrm{H}_{2}$ column densities we use the $\mathrm{CO}$ to $\mathrm{H}_{2}$ conversion factor $X_{\mathrm{CO}}$ from Bloemen et al. (1986) so,

$$
N_{\mathrm{H}_{2}}=2.8 \times 10^{20}\left(\frac{T_{b}}{\mathrm{~K} \mathrm{~km} \mathrm{~s}^{-1} \mathrm{sr}}\right) \mathrm{cm}^{-2},
$$

where $T_{B}$ is the velocity-integrated surface brightness temperature in the $\mathrm{CO} J=1-0$ map. The $X_{\mathrm{CO}}$ factor used here was chosen for consistency with Kennicutt et al. (2007), and differs from the $X_{\mathrm{CO}}=2.0 \times 10^{20}\left(\mathrm{~K} \mathrm{~km} \mathrm{~s}^{-1}\right)^{-1}$ factor used by Bigiel et al. (2008). Current uncertainties in $X_{\mathrm{CO}}$ are of the order of a factor of 2 , and the true value depends on assumptions about the dynamical state of GMCs (Blitz et al. 2007). In any case, using a different $X_{\mathrm{CO}}$ can only introduce an offset in the normalization of the SFL and should not change its observed shape.

Finally, the atomic and molecular gas surface densities are derived from the column densities using the following relations

$$
\Sigma_{\mathrm{HI}}=m_{\mathrm{H}} N_{\mathrm{HI}} \cos i
$$

$$
\Sigma_{\mathrm{H}_{2}}=2 m_{\mathrm{H}} N_{\mathrm{H}_{2}} \cos i
$$

where $m_{\mathrm{H}}$ is the hydrogen atom mass and $i=20^{\circ}$ is the inclination of NGC 5194 as measured by Tully (1974). These correspond to hydrogen gas surface densities, and should be multiplied by a factor $\sim 1.36$ to account for the mass contribution of helium and heavier elements.

\section{PHOTOIONIZATION AND SHOCK HEATING BY THE CENTRAL AGN}

The center of NGC 5194 hosts a weak active nucleus. The emission-line ratios in the narrow-line region around the AGN are consistent with those of typical Seyfert nuclei (Bradley et al. 2004, and references therein). X-ray Chandra observations show the nucleus and two extended emission components extending $\sim 15^{\prime \prime}$ north and $\sim 7^{\prime \prime}$ south of it (Terashima \& Wilson 2001). Bipolar-extended radio emission spatially coincident with the X-ray emission and a weak jet with a position angle of $158^{\circ}$ connecting the nucleus with the southern radio lobe were observed by Crane \& van der Hulst (1992) and further confirmed by Bradley et al. (2004). All the observations are consistent with the gas in the inner nuclear region $\left(r<1^{\prime \prime}\right)$ being dominantly photoionized by the central AGN, and the outer parts showing extended emission, arising from shock heating by a bipolar outflow.

For the purpose of constructing the SFL, we want to exclude regions whose main source of ionization is not UV flux coming from massive star formation. Regions in which the gas is photoionized by the AGN or shock heated by the jet will emit in $\mathrm{H} \alpha$ and mimic star formation.

In order to identify these regions we use emission-line ratio diagnostics commonly used to distinguish normal from active galaxies (Veilleux \& Osterbrock 1987; Kewley et al. 2001). Figure 6 shows the extinction-corrected [ $\left.\mathrm{N}_{\mathrm{II}}\right] \lambda 6584 / \mathrm{H} \alpha$ versus $[\mathrm{O}$ III $] \lambda 5007 / \mathrm{H} \beta$ line ratios for all the regions. The solid and dotted lines mark the theoretical threshold separating AGNs from star-forming galaxies proposed by Kewley et al. (2001) and the \pm 0.1 dex uncertainty in their modeling. To avoid the rejection of regions unaffected by AGN contamination which scatter above the threshold, we impose a double criterion. We flag as "AGN affected," all the region lying above the threshold, and at an angular distance of less than $15^{\prime \prime}$ (600 pc) from the nucleus of the galaxy. Filled triangles in Figure 6 correspond to the 17 regions complying with both criteria. Open diamonds correspond then to the 718 regions unaffected by AGN contamination we will use to construct the SFL. Note that none of these regions lie above the +0.1 dex model uncertainty dotted line, and that those lying above the threshold seem to follow the same sequence traced by the regions unaffected by AGN contamination below it. These fibers showing high line ratios but not associated with the central AGN fall in the inter-arm regions of the galaxy, and have a spectrum that is dominated by the DIG (Section 8).

Figure 7 shows a map of the $\left[\mathrm{N}_{\mathrm{II}}\right] \lambda 6584 / \mathrm{H} \alpha$ line ratio. Regions flagged as "AGN affected" are marked with black crosses. It can be seen that they have high emission-line ratios typical of AGN, and that they fall in a region which is spatially coincident with the extended radio and X-ray emission observed around the nuclei. The "AGN affected" region is elongated in a similar direction to the measured $\mathrm{PA}=158^{\circ}$ of the radio jet (Crane \& van der Hulst 1992; Bradley et al. 2004; Terashima \& Wilson 2001). Figure 7 clearly shows the enhanced line ratio in the inter-arm regions of NGC 5194. These high ratios originate in the DIG of the galaxy and are discussed in the following section. 


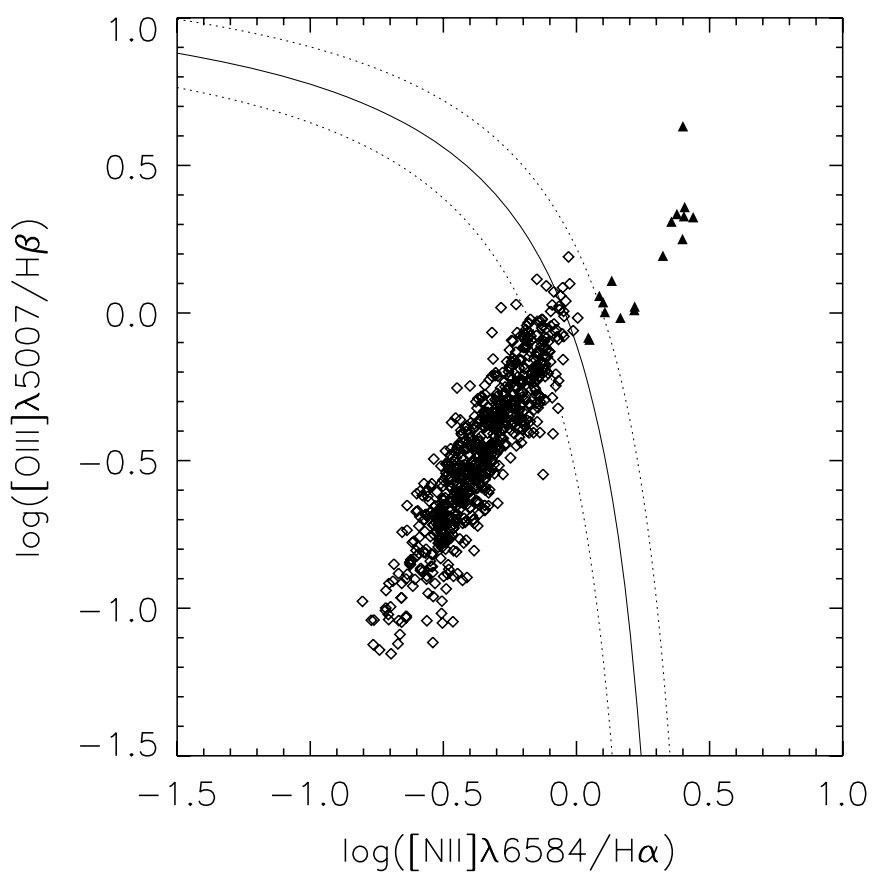

Figure 6. $[\mathrm{N}$ II $] \lambda 6584 / \mathrm{H} \alpha$ vs. $[\mathrm{O}$ III $] \lambda 5007 / \mathrm{H} \beta$ line ratio for the 735 regions. The solid line marks the theoretical threshold of Kewley et al. (2001) separating AGNs from star-forming galaxies. Dotted lines mark the \pm 0.1 dex uncertainty in the threshold modeling. The 17 regions above the threshold and having angular distances to the galaxy nucleus of $<15^{\prime \prime}$ are flagged as "AGN affected" and are shown as filled triangles. Open diamonds show the 718 regions unaffected by AGN contamination used to construct the SFL.

\section{CONTRIBUTION FROM THE DIFFUSE IONIZED GAS AND CALCULATION OF SFR SURFACE DENSITIES}

If we were to calculate $\Sigma_{\mathrm{SFR}}$ using the extinction-corrected $\mathrm{H} \alpha$ flux observed on each region, we would work under the assumption that all the emission observed in a given line of sight toward the galaxy has an origin associated with the ionizing flux coming from the localized star formation in the same region. This is not necessarily true in the presence of a diffuse ionized component in the ISM of the galaxy. The role of the DIG (a.k.a. warm ionized medium, WIM) as an important component of the ISM of star-forming disk galaxies in the local universe has been properly established during the last two decades (e.g., see reviews by Mathis 2000 and Haffner et al. 2009). The existence of a significant component of extra-planar ionized hydrogen in a galaxy requires that a fraction of the ionizing Lyman continuum photons generated in star-forming regions in the disk escapes and travels large distances of the order of kiloparsecs before ionizing neutral hydrogen at large heights above the disk. These distances are one order of magnitude larger than the Strömgren radii associated with the most massive $\mathrm{O}$ stars, and the ionizing flux is thought to escape through super-bubbles in a complex hydrogen density and ionization distribution created by supernovae, stellar winds, and large-scale ionization by $\mathrm{OB}$ associations (e.g., Dove et al. 2000).

Under these conditions a hydrogen atom emitting an $\mathrm{H} \alpha$ photon observed to come in the direction of a certain region of the galaxy is not necessarily required to have been ionized by locally produced UV photons in the same region. Hence the $\mathrm{H} \alpha$ flux measured in each region is the sum of the flux coming from $\mathrm{H}$ II regions tracing localized star formation in the disk, and a contribution from the DIG. In order to properly estimate $\Sigma_{\mathrm{SFR}}$ and the spatially resolved SFL we need to separate and subtract the DIG contribution from the observed $\mathrm{H} \alpha$ fluxes.

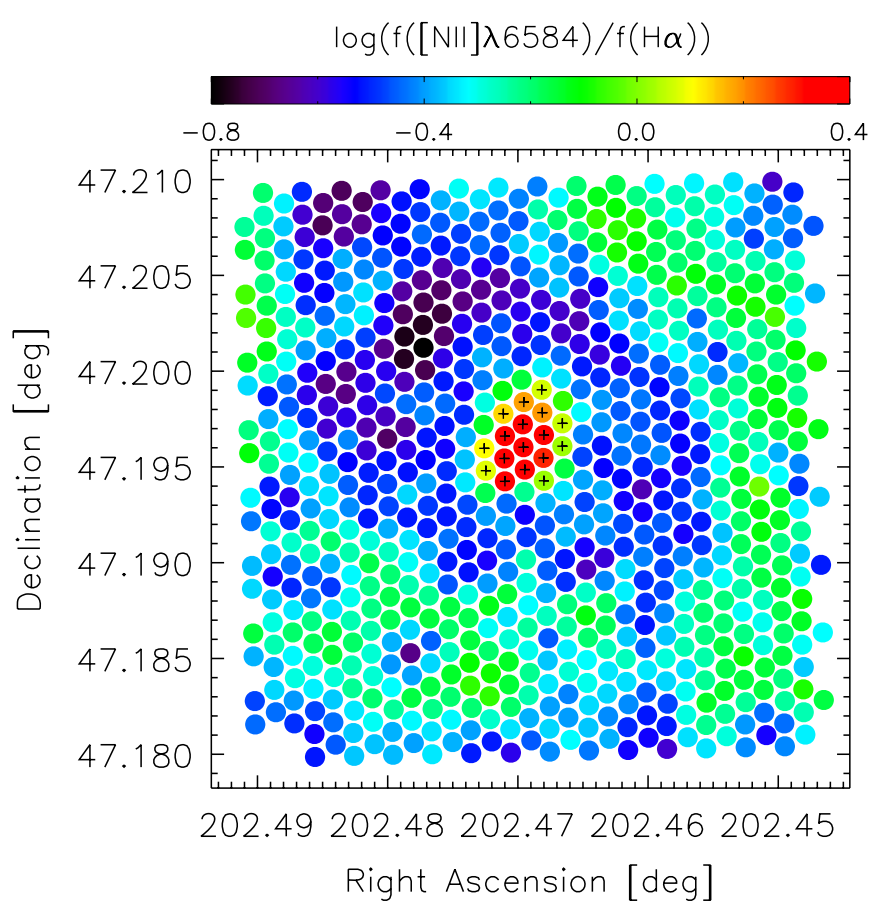

Figure 7. Map of the $\left[\mathrm{N}_{\mathrm{II}}\right] \lambda 6584 / \mathrm{H} \alpha$ emission line ratio in the central region of NGC 5194. Regions flagged as "AGN affected" are marked by black crosses. (A color version of this figure is available in the online journal.)

Low-ionization line ratios such as $\left[\mathrm{N}_{\mathrm{II}}\right] \lambda 6584 / \mathrm{H} \alpha$ and [S II] $] 6717 / \mathrm{H} \alpha$ (hereafter $[\mathrm{S}$ II] $/ \mathrm{H} \alpha$ ) are observed to be greatly enhanced in the DIG, as compared to the typical values observed in H II regions (Reynolds 1985; Hoopes \& Walterbos 2003). Recent results from The Wisconsin $\mathrm{H} \alpha$ Mapper $\left(\mathrm{WHAM}^{5}\right)$ sky survey by Madsen et al. (2006) show that $\mathrm{H}$ II regions in the MW have a typical $([\mathrm{S} \text { II }] / \mathrm{H} \alpha)_{\mathrm{H}_{\text {II }}}=0.11$ with a small $\mathrm{rms}$ scatter from region to region of only $\Delta([\mathrm{S} \text { II }] / \mathrm{H} \alpha)_{\mathrm{H} \text { II }}=0.03$. On the other hand, high galactic latitude pointings sampling the DIG component show a mean $([\mathrm{S} \mathrm{II}] / \mathrm{H} \alpha)_{\mathrm{DIG}}=0.34$, with a large scatter from pointing to pointing of $\Delta([\mathrm{S} \mathrm{II}] / \mathrm{H} \alpha)_{\mathrm{DIG}}=0.13$. Figure 8 shows a histogram of the $[\mathrm{S} \mathrm{II}] / \mathrm{H} \alpha$ line ratios taken from Madsen et al. (2006) for $\mathrm{H}$ II regions and the DIG as measured by WHAM. It can be seen that the $\left[\mathrm{S}_{\mathrm{II}}\right] / \mathrm{H} \alpha$ ratio provides a very useful tool to separate the contribution from the DIG and the disk $\mathrm{H}$ II regions in our spectra. The $[\mathrm{N}$ II $] / \mathrm{H} \alpha$ ratio, while still enhanced in the DIG as can be clearly seen in Figure 7, shows a much larger scatter both for $\mathrm{H}$ II regions and pointings toward the DIG, and does not provide such a clean separation as the $[\mathrm{S} \mathrm{II}] / \mathrm{H} \alpha$ ratio (see Figure 21 in Madsen et al. 2006).

We model the measured $\mathrm{H} \alpha$ flux of each region as the sum of a contribution from $\mathrm{H}$ II regions plus a contribution from the DIG, so

$$
\begin{aligned}
f(\mathrm{H} \alpha) & =f(\mathrm{H} \alpha)_{\mathrm{H} \text { II }}+f(\mathrm{H} \alpha)_{\mathrm{DIG}} ; \\
& =C_{\mathrm{H}_{\mathrm{II}}} f(\mathrm{H} \alpha)+C_{\mathrm{DIG}} f(\mathrm{H} \alpha),
\end{aligned}
$$

where $C_{\mathrm{H}_{\text {II }}}$ is the fraction of the total flux coming from local star-forming regions in the disk, and $C_{\mathrm{DIG}}=\left(1-C_{\mathrm{HII}}\right)$. The observed $[\mathrm{S}$ II $] / \mathrm{H} \alpha$ ratio is then given by

$$
\frac{[\mathrm{S} \text { II }]}{\mathrm{H} \alpha}=Z^{\prime}\left[C_{\mathrm{H}_{\mathrm{II}}}\left(\frac{[\mathrm{S} \mathrm{II}]}{\mathrm{H} \alpha}\right)_{\mathrm{H} \text { II }}+C_{\mathrm{DIG}}\left(\frac{[\mathrm{S} \mathrm{II}]}{\mathrm{H} \alpha}\right)_{\mathrm{DIG}}\right],
$$

\footnotetext{
5 http://www.astro.wisc.edu/wham/
} 


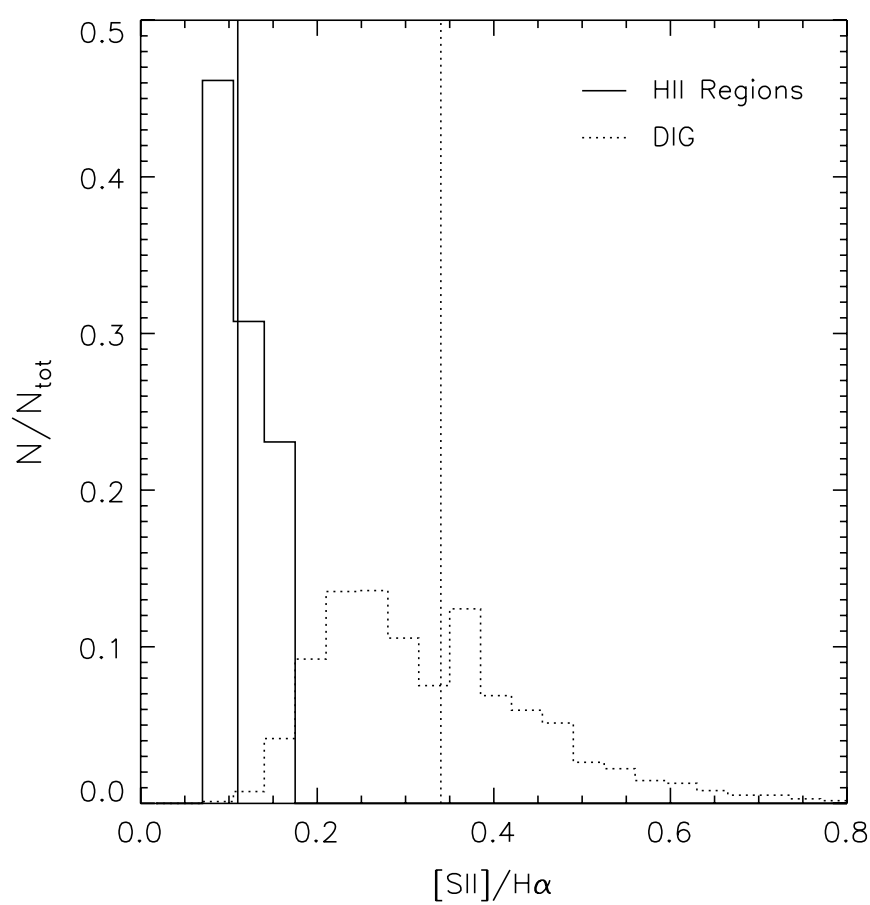

Figure 8. Histogram of the [S II] $/ \mathrm{H} \alpha$ of $\mathrm{H}$ II regions (solid) and pointings toward DIG (dotted) in the MW as measured by WHAM (Madsen et al. 2006). Vertical lines mark the mean values for the two distributions.

where $Z^{\prime}=Z / Z_{\mathrm{MW}}$ is the metallicity of NGC 5194 normalized to the MW value. Figure 9 shows the observed [S II]/H $\alpha$ ratio as a function of extinction-corrected $\mathrm{H} \alpha$ flux. The left axis shows $C_{\mathrm{H} \text { II }}$ calculated assuming a value of $Z^{\prime}=1.0 / 1.5$. Bresolin et al. (2004) measured the oxygen and sulfur abundance gradient in NGC 5194 using multi-object spectroscopy of $10 \mathrm{H}$ II regions spanning a large range in radii. Integrating his best-fitted oxygen abundance gradient out to a radius of $4.1 \mathrm{kpc}$ provides a mean $12+\log (\mathrm{O} / \mathrm{H})=8.68$, which is 1.55 times lower than the solar oxygen abundance measured by Grevesse et al. (1996). Although a large scatter is observed in the literature for both the solar oxygen abundance and the oxygen abundance in MW H II regions (Grevesse et al. 1996; Allende Prieto et al. 2001; Shaver et al. 1983; Deharveng et al. 2000), it can be seen in Figure 9 that using a factor of 1.5 implies that the brightest $\mathrm{H} \alpha$ emitting regions in NGC 5194 are completely dominated by emission from $\mathrm{H}$ II regions in the disk, having $C_{\mathrm{H}_{\text {II }}} \sim 1$ with a scatter that is consistent with the intrinsic scatter of 0.03 measured in the MW by Madsen et al. (2006). These brightest regions trace the spiral structure of the galaxy and their spectra are expected to be $\mathrm{H}$ II region dominated since on high star formation regions the disk should outshine the DIG by many orders of magnitude.

There is a clear correlation between $C_{\mathrm{H} \text { II }}$ and the $\mathrm{H} \alpha$ flux. The observed trend is consistent with the DIG dominating the spectrum of fainter $\mathrm{H} \alpha$ regions, and the $\mathrm{H}$ II regions in the disk outshining the DIG in the brightest ones. The scatter is large mostly because of intrinsic scatter in the line ratio (see Figure 8). In order to compute a robust DIG correction, we fit the $C_{\mathrm{H} \text { II }}$ values using the simple functional form

$$
C_{\mathrm{H} \text { II }}=1.0-\frac{f_{0}}{f(\mathrm{H} \alpha)} ; \quad\left(\text { for } f(\mathrm{H} \alpha)>f_{0}\right),
$$

where $f_{0}=3.69 \times 10^{-15} \mathrm{erg} \mathrm{s}^{-1} \mathrm{~cm}^{-2}$ is the flux at which the DIG contributes $100 \%$ of the emission, and hence $C_{\mathrm{HII}}=0$ for $f(\mathrm{H} \alpha) \leqslant f_{0}$. The correction is shown as the red solid line in

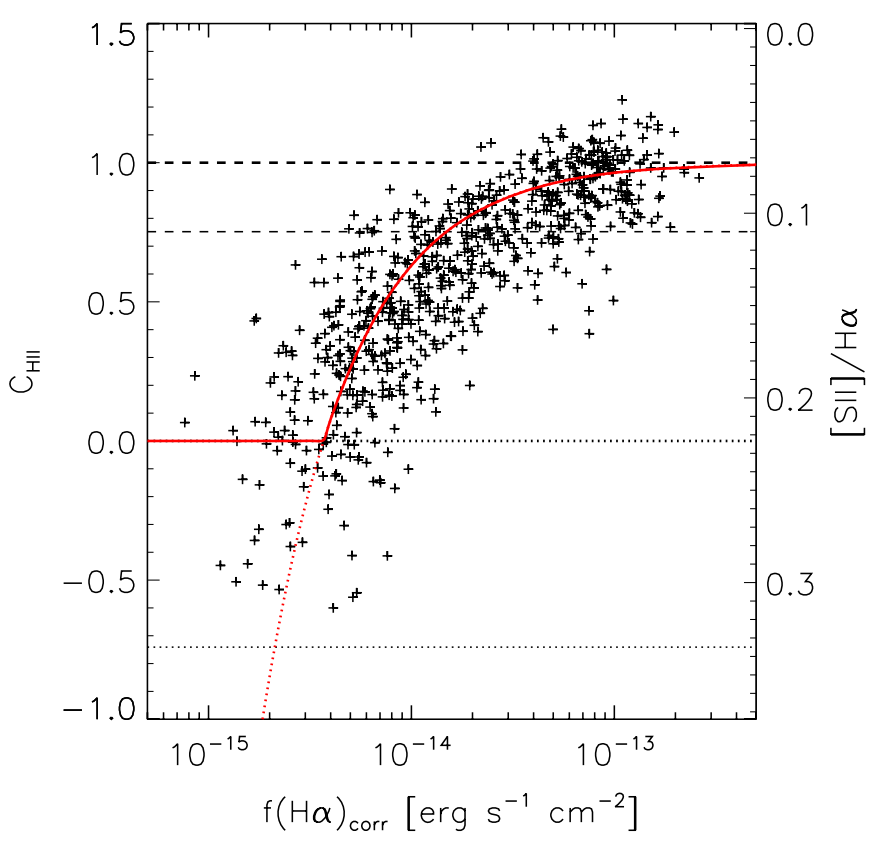

Figure 9. Observed $[\mathrm{S} \mathrm{II}] / \mathrm{H} \alpha$ emission line ratio for the 718 regions unaffected by AGN contamination. The thin dashed and dotted lines show the mean ratio observed in $\mathrm{H}$ II regions and pointings toward the DIG in the MW, respectively. The thick dashed and dotted lines show the former ratios scaled down by a factor $Z^{\prime}=1.0 / 1.5$. The left axis shows the fraction of the flux coming from $\mathrm{H}$ II regions in the disk given by Equation (8). The solid red curve shows the DIG correction applied to the data given by Equation (9), and the continuation of the function to fluxes lower than $f_{0}$ is marked by the dotted red line.

(A color version of this figure is available in the online journal.)

Figure 9. We multiply the extinction-corrected $\mathrm{H} \alpha$ fluxes by the above correction factor in order to remove any contribution from the DIG in NGC 5194. It is worth noting that using Equation (9) to remove the DIG is equivalent to subtracting a constant DIG flux value $f_{0}$ for all regions with $f(\mathrm{H} \alpha)>f_{0}$ (the large majority of the regions). Hence, the line ratio distribution presented in Figure 9 is very well fitted by a flat DIG component.

Figure 10 presents maps of the extinction-corrected $\mathrm{H} \alpha$ emission line flux before and after the DIG correction is applied. It can be seen clearly how the $\mathrm{H} \alpha$ emission traces the spiral pattern of star formation. The correction leaves the $\mathrm{H} \alpha$ flux coming from the brightest star-forming regions practically unchanged, while removing the contribution from the DIG which dominates the observed spectrum in the inter-arm regions of the galaxy. The latter can also be appreciated in Figure 7, which shows an enhanced [N $\left.{ }_{\text {II }}\right] / \mathrm{H} \alpha$ ratio typical of the DIG in the inter-arm regions, and normal $\mathrm{H}$ il region ratios throughout the spiral arms.

Integrating over the complete observed area, the DIG contributes only $11 \%$ of the total $\mathrm{H} \alpha$ flux. Previous photometric measurements of the diffuse ionized fraction in nearby spiral galaxies, including NGC 5194, yield median diffuse fractions of $\sim 50 \%$ (e.g., Ferguson et al. 1996; Hoopes et al. 1996; Greenawalt et al. 1998; Thilker et al. 2002; Oey et al. 2007). These studies are performed either by masking of $\mathrm{H}$ II regions or by discrete $\mathrm{H}$ II region photometry in $\mathrm{H} \alpha$ narrowband images. Although it will be seen in Section 10 that the assumption of a constant $[\mathrm{N}$ II $] / \mathrm{H} \alpha$ ratio throughout the galaxy used to correct the narrow-band images in all the above studies can introduce overestimations of the DIG $\mathrm{H} \alpha$ brightness of up to $40 \%$, this effect is small, and cannot account for the difference between our diffuse fraction and the typical values found 

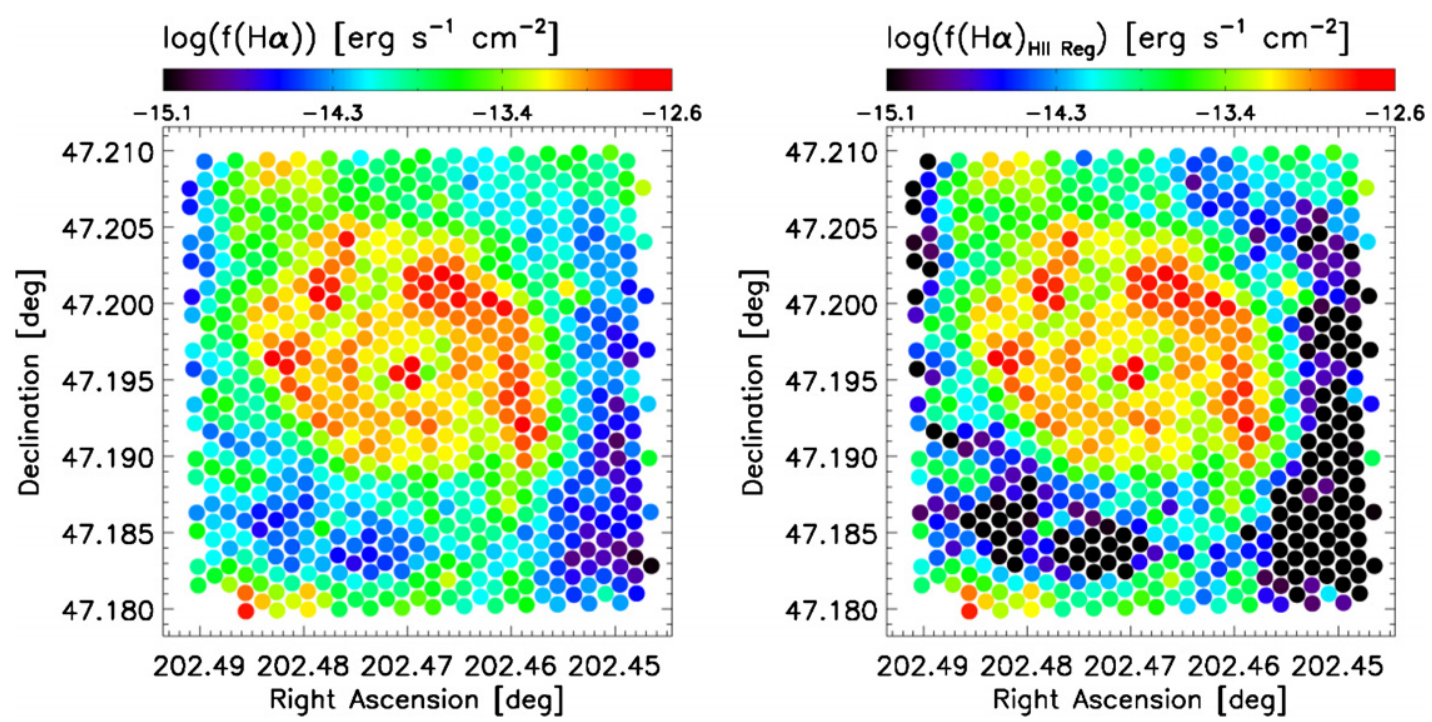

Figure 10. Left: map of the extinction-corrected $\mathrm{H} \alpha$ nebular emission flux in the central $4.1 \times 4.1 \mathrm{kpc}^{2}$ of NGC 5194. Right: same map after removing the DIG contribution to the $\mathrm{H} \alpha$ emission line flux, that is, showing only the flux coming from H II regions in the disk of NGC 5194.

(A color version of this figure is available in the online journal.)

in the literature. The difference is most likely due to the fact that our observations are limited to the highly molecular, and hence strongly star-forming central part of the galaxy. Our measured diffuse ionized fraction is then only a lower limit to the DIG contribution over the whole galaxy, since at larger radii the relative contribution from $\mathrm{H}$ II regions is expected to significantly decrease. Though the DIG contribution to the integrated $\mathrm{H} \alpha$ luminosity in the central region of NGC 5194 could be small, on the small scales sampled by the VIRUS-P fibers the DIG can account for $100 \%$ of the observed $\mathrm{H} \alpha$ flux, especially in between the spiral arms where $\mathrm{H}$ II regions are rare. Given the clear dependence of the above correction with the $\mathrm{H} \alpha$ flux, failing to correct for this effect introduces a bias in the SFL toward shallower slopes.

The corrected $\mathrm{H} \alpha$ emission-line fluxes are transformed into $\mathrm{H} \alpha$ luminosities using the assumed distance to NGC 5194 of 8.2 Mpc. Since the DIG is suspected to arise from UV photons escaping star-forming regions in the disk, not accounting for these photons should introduce a systematic underestimation of the SFR. The challenge resides in our inability to tell from where in the disk these UV photons come from. To ameliorate this problem, we scale the $\mathrm{H} \alpha$ luminosities by a factor of 1.11 , which is equivalent to assuming that the UV photons ionizing the DIG were originated in the star-forming regions in the disk proportionally to their intrinsic UV luminosities. These scaled luminosities $\left(L_{\text {corr }}(\mathrm{H} \alpha)\right)$ are used to estimate the SFR for each of the 718 regions. We use the calibration presented in Kennicutt (1998a), for which the SFR is given by

$$
\operatorname{SFR}\left[M_{\odot} \mathrm{yr}^{-1}\right]=7.9 \times 10^{-42} L_{\text {corr }}(\mathrm{H} \alpha)\left(\mathrm{erg} \mathrm{s}^{-1}\right) .
$$

The above calibration assumes a Salpeter IMF over the range of stellar masses 0.1-100 $M_{\odot}$. To convert to the Kroupa-type two-component IMF used in Bigiel et al. (2008), the SFR must be multiplied by a factor of 0.63 .

The SFRs for individual regions are then converted to SFR surface densities $\left(\Sigma_{\mathrm{SFR}}\right)$. Following Kennicutt et al. (2007), we divide the SFR by the projected area on the sky of the 4."3 (172 pc) diameter regions sampled by each fiber on the IFU, and multiply it by a factor of $\cos \left(20^{\circ}\right)$ to account for the inclination of NGC 5194 (Tully 1974).

\section{THE SPATIALLY RESOLVED STAR FORMATION LAW}

The observed relations between $\Sigma_{\mathrm{SFR}}$ and the gas surface densities of different components of the ISM $\left(\Sigma_{\mathrm{H}_{\mathrm{I}}}, \Sigma_{\mathrm{H}_{2}}\right.$, and $\Sigma_{\mathrm{H}_{\mathrm{I}+\mathrm{H}_{2}}}$ ) are presented in Figures 11-13. Error bars in gas surface densities correspond to the $1 \sigma$ uncertainties given in Sections 4.1 and 4.2. Error bars in the SFR surface density include a series of uncertainties that we proceed to describe. First we consider the uncertainty in the observed $\mathrm{H} \alpha$ fluxes. This comes from the fitting of the $\mathrm{H} \alpha$ line described in Section 5.2, which was performed considering the observational error in the spectrum (obtained from the error maps described in Section 3). Second, the uncertainty in the dust extinction correction is included by propagating the fitting errors of the observed $\mathrm{H} \alpha$ and $\mathrm{H} \beta$ fluxes through Equation (2). Finally, in order to account for the error associated with the DIG correction, we introduce a $20 \%$ uncertainty in $\Sigma_{\mathrm{SFR}}$, consistent with the median scatter of the points in Figure 9 around the correction used. All these uncertainties are summed in quadrature to account for the error bars in $\Sigma_{\mathrm{SFR}}$. We do not consider errors in the flux calibration which are expected to be of $\sim 5 \%$, nor in the $\mathrm{CO}$ to $\mathrm{H}_{2}$ conversion factor. The later is currently highly uncertain and might change by up to a factor of 2 depending on assumptions about the dynamical state of GMCs (Blitz et al. 2007). In any case, these two sources of systematic errors enter the SFL as multiplicative factors. Hence, they can only introduce a bias in the normalization of the SFL, and should not affect the fitted values of the slope and the intrinsic scatter.

From Figure 11 it is clear that $\Sigma_{\mathrm{SFR}}$ shows a very poor correlation with $\Sigma_{\mathrm{HI}}$, since regions having similar atomic gas budgets can have star formation activities that differ by more than 3 orders of magnitude. We observe an evident saturation in the atomic gas surface density at $\Sigma_{\mathrm{H}_{\mathrm{I}}} \approx 10 M_{\odot} \mathrm{pc}^{-2}$. Also, there is a slight inversion in the sense of the correlation at high $\Sigma_{\mathrm{SFR}}$, associated with the central part of the galaxy due to the presence of a minimum in the $\mathrm{H}$ I profile (Bigiel et al. 2008). These $\mathrm{H} \mathrm{I}$ "holes" are common in the centers of spiral galaxies, and in them the ISM is fully dominated by molecular hydrogen while the atomic gas is almost completely depleted. The saturation at $10 M_{\odot} \mathrm{pc}^{-2}$ has been previously observed by Wong \& Blitz (2002) using azimuthally averaged data, and further confirmed 


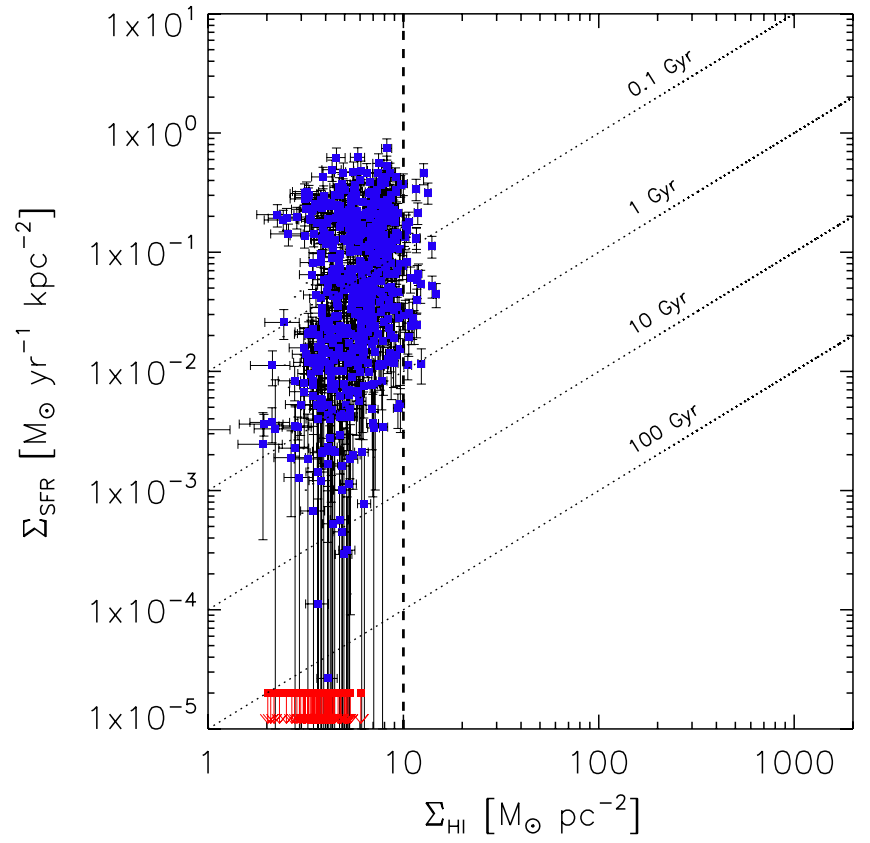

Figure 11. Atomic gas surface density vs. SFR surface density for the 718 regions unaffected by AGN contamination. Upper limits in $\Sigma_{\text {SFR }}$ correspond to regions with $C_{\mathrm{H} \text { II }}=0$. The vertical dashed line marks the $\mathrm{HI}_{\mathrm{I}}$ to $\mathrm{H}_{2}$ transition threshold at $10 M_{\odot} \mathrm{pc}^{-2}$. The diagonal dotted lines correspond to constant depletion timescales $\tau=\mathrm{SFE}^{-1}$ of $0.1,1,10$, and $100 \mathrm{Gyr}$.

(A color version of this figure is available in the online journal.)

to be a widespread phenomena in normal spirals by Bigiel et al. (2008) using two-dimensional spatially resolved measurements. It is thought to be related to a threshold in surface density at which a phase transition from atomic to molecular gas occurs in the ISM (Krumholz et al. 2009a). Given the lack of correlation between $\Sigma_{\mathrm{H}_{\mathrm{I}}}$ and $\Sigma_{\mathrm{SFR}}$, we do not attempt to fit a atomic gas SFL. We restrict our analysis to the modeling of the molecular and total gas correlations with the star formation activity. These correlations are usually well described by a power-law function (Schmidt 1959; Kennicutt 1998b).

It has been established that the observed rms dispersion about a power law in these SFLs is much larger than the observational uncertainties (Kennicutt 1998b; Kennicutt et al. 2007), implying the existence of significant intrinsic scatter of physical origin in the relations. However, previous works have not introduced this intrinsic scatter into the parameterization of the SFL, and authors restrict themselves to measure the scatter after fitting a power law to the data. In this work, we incorporate the intrinsic scatter in the SFL, which we parameterize as

$$
\frac{\Sigma_{\mathrm{SFR}}}{1 M_{\odot} \mathrm{yr}^{-1} \mathrm{kpc}^{-2}}=A\left(\frac{\Sigma_{\mathrm{gas}}}{100 M_{\odot} \mathrm{pc}^{-2}}\right)^{N} \times 10^{\mathcal{N}(0, \epsilon),}
$$

where $A$ is the normalization factor, $N$ is the slope, and $\mathcal{N}(0, \epsilon)$ is a logarithmic deviation from the power law, drawn from a normal distribution with zero mean and standard deviation $\epsilon$. The value of $\epsilon$ corresponds to the intrinsic scatter of the SFL in logarithmic space. The factor $10^{\mathcal{N}(0, \epsilon)}$ can be interpreted as changes of physical origin in the SFE for different regions. We chose a pivot value for the normalization of $100 M_{\odot} \mathrm{pc}^{-2}$, which is roughly at the center of the distribution of measured $\Sigma_{\text {gas }}$ values, in order to minimize the covariance between the slope and the normalization. When comparing the normalization factors derived here with other fits found in the literature, this must be taken into account. Most works quote normalizations

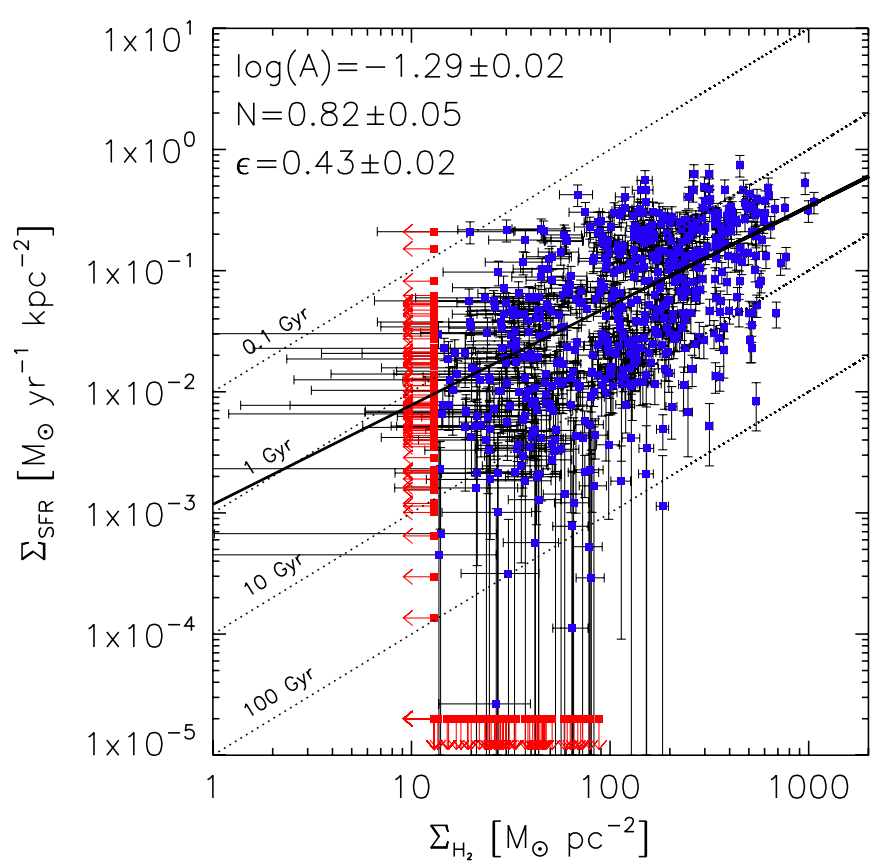

Figure 12. Molecular gas surface density vs. SFR surface density for the 718 regions unaffected by AGN contamination. Upper limits in $\Sigma_{\text {SFR }}$ correspond to regions with $C_{\mathrm{H} \text { II }}=0$. Upper limits in $\Sigma_{\mathrm{H}_{2}}$ correspond to regions with nondetection in $\mathrm{CO}$ at the $1 \sigma$ level. The diagonal dotted lines correspond to constant depletion timescales $\tau=\mathrm{SFE}^{-1}$ of $0.1,1,10$, and $100 \mathrm{Gyr}$. Also shown is the best-fitted power law from the MC method (black solid line), and the best-fitted parameters.

(A color version of this figure is available in the online journal.)

at $1 M_{\odot} \mathrm{pc}^{-2}$, while Bigiel et al. (2008) quote normalizations at $10 M_{\odot} \mathrm{pc}^{-2}$.

Previous measurements of the spatially resolved SFL use different algorithms to fit a power law to the data. Usually a linear regression in logarithmic space is performed, but methods differ in the treatment of error bars. Kennicutt et al. (2007) used a FITEXY algorithm (Press et al. 1989), which has the advantage of incorporating errors in both the ordinate and abscissa coordinates, although errors must be assumed to be symmetric in logarithmic space, which is not always the case. Bigiel et al. (2008) used an ordinary least-squares (OLS) bisector method (Isobe et al. 1990) giving the same weighting to every data point. Both methods have the disadvantage of not being able to incorporate upper limits in the minimization. Our data are mainly limited by the sensitivity of the $\mathrm{CO}$ intensity maps as can be seen in Figure 12, where 93 of the 718 regions unaffected by AGN contamination are undetected in $\mathrm{CO}$ and hence we can only provide upper limits for their molecular gas surface densities. This is also the case in the works mentioned above. As will be seen in Section 11, these upper limits contain important information regarding the slope of the spatially resolved SFL, and neglecting them biases the fits toward steeper slopes. We introduce and use a new method for fitting the SFL which is not affected by the above issues.

\subsection{The Fitting Method}

To fit our data we use a Monte Carlo (MC) approach combined with two-dimensional distribution comparison techniques commonly used in color-magnitude diagram (CMD) fitting (Dolphin et al. 2001). Our method allows us to include the regions not detected in the CO map (including those with negative measured fluxes), incorporate the intrinsic scatter in the SFL as a free 


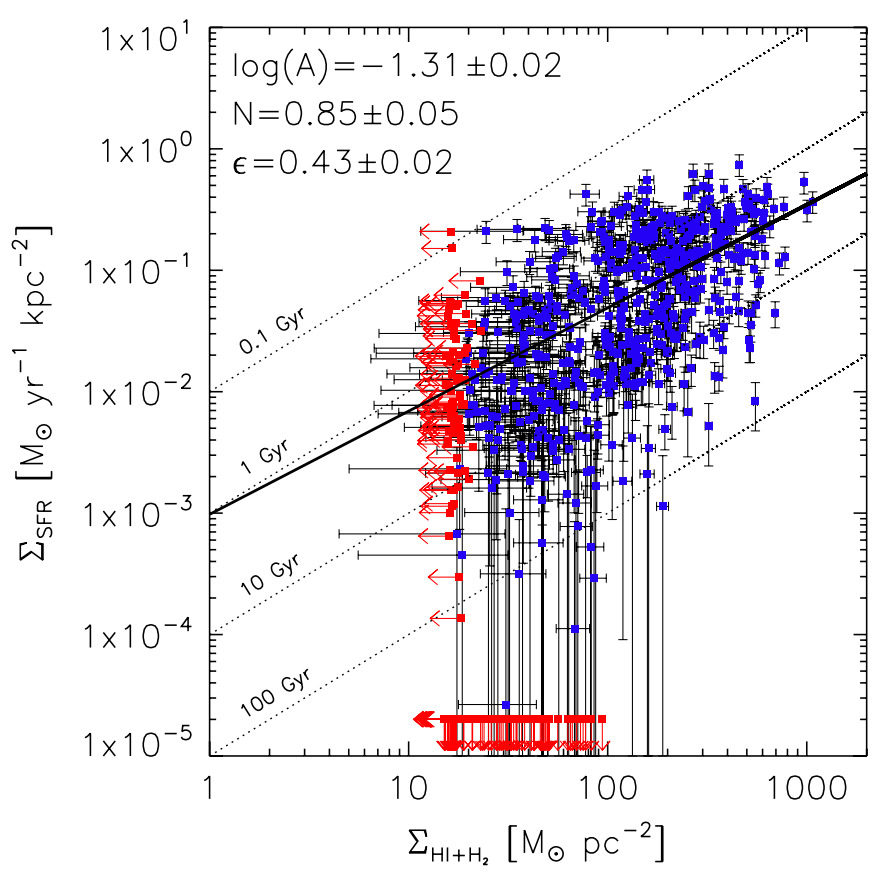

Figure 13. Total gas surface density vs. SFR surface density for the 718 regions unaffected by AGN contamination. Upper limits in $\Sigma_{\mathrm{SFR}}$ correspond to regions with $C_{\mathrm{H} \text { II }}=0$. Upper limits in $\Sigma_{\mathrm{H}_{\mathrm{I}} \mathrm{H}_{2}}$ correspond to regions with non-detection in $\mathrm{CO}$ at the $1 \sigma$ level. The diagonal dotted lines correspond to constant depletion timescales $\tau=\mathrm{SFE}^{-1}$ of $0.1,1,10$, and $100 \mathrm{Gyr}$. Also shown is the best-fitted power law from the MC method (black solid line), and the best-fitted parameters. (A color version of this figure is available in the online journal.)

parameter, and perform the fitting in linear space, avoiding the assumption of log-normal symmetric errors. In the following, we describe our fitting method.

For any given set of parameters $\{A, N, \epsilon\}$, we generate $200 \mathrm{MC}$ realizations of the data. To create each realization, we take the measured values of $\Sigma_{\text {gas }}$ as the true values and calculate the corresponding true $\Sigma_{\text {SFR }}$ using Equation (11), drawing a new value from $\mathcal{N}(0, \epsilon)$ for each point in order to introduce the intrinsic scatter. Regions for which we measure negative CO fluxes are assumed to have $\Sigma_{\text {gas }}=\Sigma_{\mathrm{SFR}}=0$. In order to account for observational errors, data points are then offset in $\Sigma_{\mathrm{SFR}}$ and $\Sigma_{\text {gas }}$ by random quantities given by the observed measurement error for each data point. The uncertainty in $\Sigma_{\mathrm{SFR}}$ is largely dominated by the errors introduced in the dust extinction and DIG corrections. Since both corrections are multiplicative, we apply the random offsets as multiplicative factor drawn from a $\mathcal{N}\left(1, \sigma\left(\Sigma_{\mathrm{SFR}}\right) / \Sigma_{\mathrm{SFR}}\right)$ distribution. On the other hand, the error in $\Sigma_{\text {gas }}$ is dominated by systematic offsets introduced during the combination and calibration of interferometric data. Accordingly, the random offsets in $\Sigma_{\text {gas }}$ are introduced in an additive manner, using values drawn from a $\mathcal{N}\left(0, \sigma\left(\Sigma_{\text {gas }}\right)\right)$ distribution. It is important to note that while for plotting purposes, Figures 11-13 show upper limits in $\Sigma_{\text {gas }}$ and $\Sigma_{\text {SFR }}$, in the fitting procedure the measured values of these data points are used together with their usually large error bars.

Having the observed data points and the large collection of realizations of the data coming from the model, we need to compare the distribution of points in the $\Sigma_{\text {gas }}-\Sigma_{\mathrm{SFR}}$ plane in order to assess how well the model fits the data given the assumed parameters. To do so, we define a grid on the $\Sigma_{\text {gas }}-\Sigma_{\text {SFR }}$ plane and count the number of data points falling on each grid element both in the data and in the 200 realizations. This method is adapted from Dolphin et al. (2001), and it is the equivalent to the construction of Hess diagrams used in CMD fitting. The grid covers all the observed data points, has a resolution of $\Delta \Sigma_{\mathrm{gas}}=156 M_{\odot} \mathrm{pc}^{-2}$ and of $\Delta \Sigma_{\mathrm{SFR}}=0.11 M_{\odot} \mathrm{yr}^{-1} \mathrm{kpc}^{-2}$, and is shown in the left panel of Figure 14. A single extra grid element containing all the points in the $\mathrm{MC}$ realizations falling outside the grid and zero observed data points is also included in the calculations below.

We average the number of points in each grid element for the $200 \mathrm{MC}$ realizations and call this "the model." In order to compare the model to the data we compute a $\chi^{2}$ statistic of the following form:

$$
\chi^{2}=\sum_{i} \frac{\left(N_{i}-M_{i}\right)^{2}}{M_{i}},
$$

where the sum is over all the grid elements in the $\Sigma_{\mathrm{gas}}-\Sigma_{\mathrm{SFR}}$ plane, $N_{i}$ is the number of observed data points, and $M_{i}$ is the number of model data points in the grid element $i$. We sample a large three-dimensional grid in parameter space with a resolution of $\Delta \log (A)=0.018, \Delta N=0.036$, and $\Delta \epsilon=0.011$, centered around our best initial guesses for the different SFLs, and compute $\chi^{2}$ for every combination of parameters in the cube.

To exemplify our method, the left panel in Figure 14 shows the observed molecular SFL in linear space, together with the best-fitted MC model. Overlaid are all the grid elements, colorcoded according with the density of points inside each of them. The top central panel shows the number of points in each grid element in the model versus the data for the best-fitted model, in this plot, deviations from the dashed line contribute to the $\chi^{2}$ statistic. Also shown is the $\chi^{2}$ for each parameter, marginalized over the other two. The best-fitted value for each parameter is obtained by fitting a quadratic function to the minimum $\chi^{2}$ at each parameter value sampled. Uncertainties at the $1 \sigma, 2 \sigma$, and $3 \sigma$ levels are also shown in the plots. Note that the sampled set of parameters showing the minimum $\chi^{2}$ is always within $1 \sigma$ of the best-fitted value deduced from the quadratic function fitting.

Thorough testing of the fitting method was carried out. The number of $\mathrm{MC}$ simulations is high enough for consecutive runs of the algorithm on the same data to produce best-fitted values for the parameters that show a scatter of less than $0.1 \sigma$. The best-fitted parameters are somewhat sensitive to the chosen grid spacing in the linear $\Sigma_{\text {gas }}-\Sigma_{\text {SFR }}$ plane. Fitting of artificially generated data sets drawn from known parameters showed the grid resolution we use to be the best at recovering the intrinsic parameters with deviations from the true values of less than $0.5 \sigma$.

\subsection{Fits to the Molecular and Total Gas Star Formation Laws}

We applied our method to fit the observed SFL in both molecular gas and total gas. The best-fitted SFLs are shown as solid lines in Figures 12 and 13, where the best-fitted parameters are also reported. For the molecular gas SFL we measure a slope $N=0.82 \pm 0.05$, an amplitude $A=10^{-1.29 \pm 0.02}$, and an intrinsic scatter $\epsilon=0.43 \pm 0.02$ dex. In the central part of NCG 5194 we sample a density regime in which the ISM is almost fully molecular; hence the total gas SFL closely follows the molecular SFL and shows very similar bestfitted parameters. For the total gas SFL we obtain a slope $N=0.85 \pm 0.05$, an amplitude $A=10^{-1.31 \pm 0.02}$, and an intrinsic scatter $\epsilon=0.43 \pm 0.02$ dex.

Of great interest is the large intrinsic scatter observed in the SFL. A logarithmic scatter of 0.43 dex implies that the SFR 

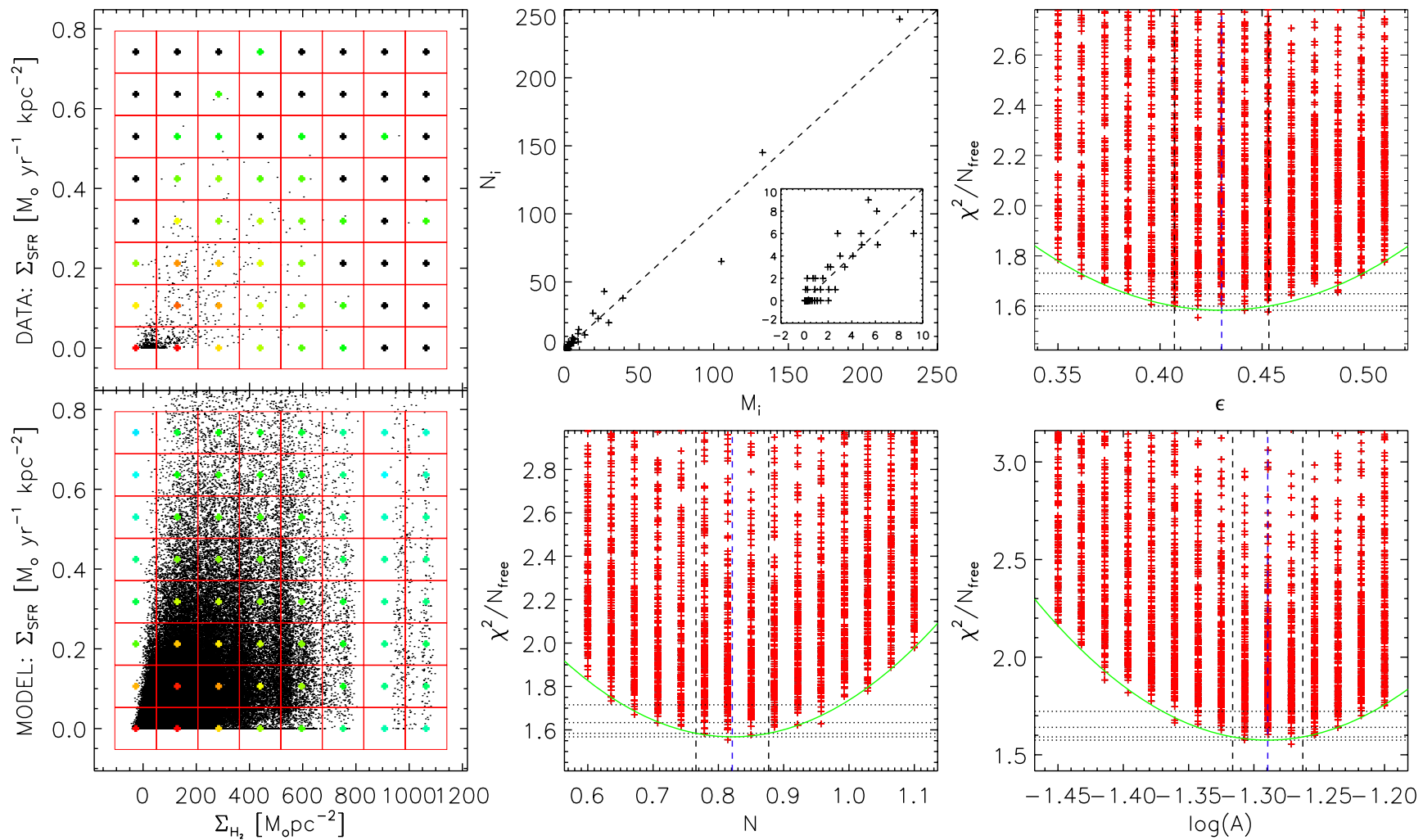

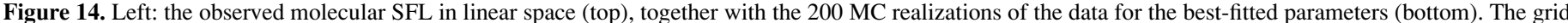

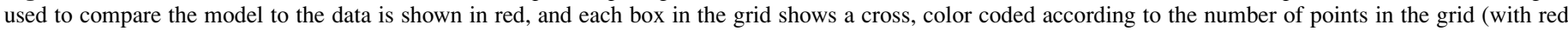

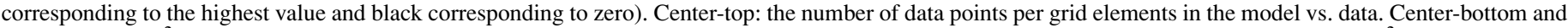

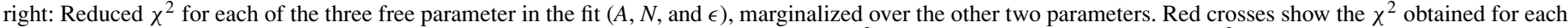

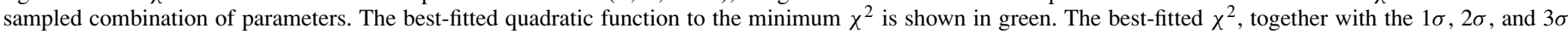
levels are shown as horizontal dotted lines. The blue and black vertical dashed lines mark the best-fitted parameter and its $1 \sigma$ uncertainty, respectively.

(A color version of this figure is available in the online journal.)

in regions having the same molecular gas surface density can vary roughly by a factor of $\sim 3$. This is very important to keep in mind when using the SFL as a star formation recipe in theoretical models of galaxy formation and evolution. Results from this type of modeling should be interpreted in an statistical sense, and we must always remind ourselves that SFRs predicted for single objects can be off by these large factors. The bottom left panel of Figure 14 is an striking reminder of the limitations involved in the use of SFLs as star formation recipes in analytical and semi-analytical models. The large scatter observed is indicative of the existence of other parameters, besides the availability of molecular gas, which are important in setting the SFR.

As will be discussed in Section 11, the fact that we measure a slightly sub-linear SFL is consistent with recent results by Bigiel et al. (2008) and Leroy et al. (2008), as well as with recent theoretical modeling by Krumholz et al. (2009b), but in disagreement with the significantly super-linear molecular and total gas SFLs measured in NGC 5194 by Kennicutt et al. (2007). Our results imply depletion times for the molecular gas of $\tau \approx 2 \mathrm{Gyr}$, which is roughly a factor of $\sim 100$ longer than the typical free fall time of GMCs (McKee 1999). These low efficiencies, of the order of $1 \%$ per free-fall time, are observed in a large range of spatial scales and densities in different objects. It is seen all the way from HCN emitting clumps, infrared dark clouds, and GMCs in the MW to the molecular ISM in large scales in normal spiral galaxies and starburst, and is consistent with models in which star formation is regulated by supersonic turbulence in GMCs, induced by feedback from star formation itself (Evans et al. 2009; Krumholz \& Tan 2007).

\section{BALMER ABSORPTION AND THE [N II]/H $\alpha$ RATIO, IMPLICATIONS FOR NARROW-BAND IMAGING}

Narrow-band imaging is the most widely used method for conducting spatially resolved measurements of the $\mathrm{H} \alpha$ emission line in nearby galaxies. Images taken with a narrow-band filter centered at $\mathrm{H} \alpha$, and either a broad-band or off-line narrowband, are subtracted in order to remove the continuum in the on-line bandpass. The excess flux in the on-line narrowband is expected to map the nebular emission. Narrow-band filters have typical FWHMs of $\sim 70 \AA$, and hence suffer from contamination from the $[\mathrm{N}$ II $] \lambda \lambda, 6548,6584$ doublet. Also, narrow-band techniques cannot directly separate the nebular emission from the underlying photospheric absorption $\mathrm{H} \alpha$. Corrections to account for these two factors are usually applied.

In order to correct for the underlying absorption, the continuum image is usually scaled before subtraction so selected regions in the galaxy, which are a priori expected to be free of $\mathrm{H} \alpha$ emission, show zero flux in the subtracted image. This is equivalent to correcting for a constant $\mathrm{H} \alpha$ absorption equivalent width (EW) across the galaxy (assuming that the continuum level was reliably estimated, which might not be the case when broadbands are used instead of off-line narrow bands, since the spectral slope of the stellar continuum can vary significantly across the galaxy). The [N II] contamination is usually taken out by assuming a constant $[\mathrm{N} \mathrm{II}] / \mathrm{H} \alpha$ ratio across the whole galaxy, which together with the relative filter transmission at the wavelengths of the three lines, is used to compute a correction factor which is used to scale down the observed continuum subtracted 


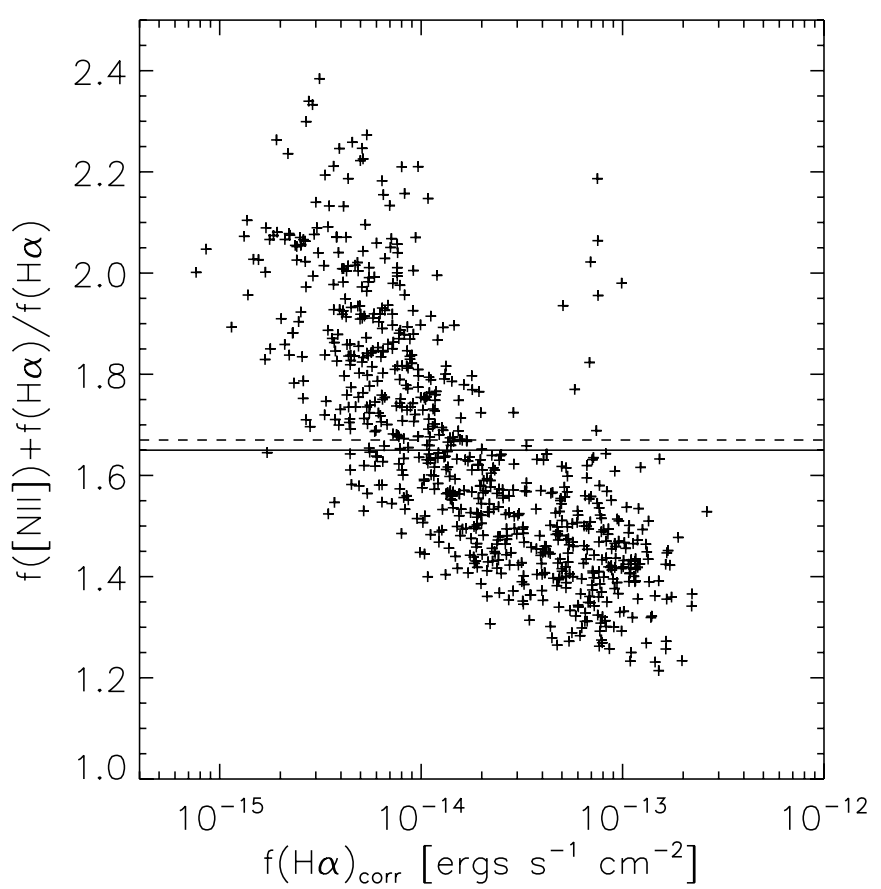

Figure 15. $([\mathrm{N}$ II $] \lambda 6548+[\mathrm{N}$ II $] \lambda 6584+\mathrm{H} \alpha) / \mathrm{H} \alpha$ ratio as a function of extinctioncorrected $\mathrm{H} \alpha$ flux for the 718 regions under study. The solid line marks the observed mean value of 1.65 . The dashed line marks the 1.67 value expected by assuming the line ratio of $[\mathrm{N}$ II $] \lambda 6584 / \mathrm{H} \alpha=0.5$ and $[\mathrm{N}$ II] $]$ 6548/ $[\mathrm{N}$ II $] \lambda 6584=0.335$.

narrow-band fluxes in order to remove the [N II] contribution. Integral-field spectroscopy is free of these two effects, since both the [N $\mathrm{NI}]$ lines and the photospheric $\mathrm{H} \alpha$ absorption can be clearly separated from the $\mathrm{H} \alpha$ emission (see Figure 3). Thus, our observations provide an important check on the validity of the corrections typically applied in narrow-band studies, and the biases introduced by them.

For the $[\mathrm{N}$ II] correction, line ratios of $[\mathrm{N}$ II $] \lambda 6584 / \mathrm{H} \alpha=0.5$ and $\left[\mathrm{N}_{\mathrm{II}}\right] \lambda 6548 /\left[\mathrm{N}_{\mathrm{II}}\right] \lambda 6584=0.335$ are typically assumed (Calzetti et al. 2005). Based on these ratios, a perfect $\mathrm{H} \alpha$ filter (i.e., one with a constant transmission across the three lines) would measure a flux that is a factor of 1.67 higher than the $\mathrm{H} \alpha$ flux. Figure 15 shows the ([N II $] \lambda 6548+[\mathrm{N}$ II $] \lambda 6584+\mathrm{H} \alpha) /$ $\mathrm{H} \alpha$ ratio as a function of the extinction-corrected $\mathrm{H} \alpha$ flux, as measured in the VIRUS-P spectra of all 718 star-forming regions. Although we measure a mean value of 1.65 (solid line), in good agreement with the predictions from the above line ratios (dashed line), it can be seen that the correction factor is a strong function of the $\mathrm{H} \alpha$ flux. The fact that we observe an increasing [N II] $/ \mathrm{H} \alpha$ ratio as we go to fainter $\mathrm{H} \alpha$ fluxes is consistent with the nebular emission in the faintest parts of the galaxy (mainly the inter-arm regions) being dominated by the DIG component of the ISM (see Figure 7 and Section 8).

The observed line ratios imply that assuming a constant [N II]/ $\mathrm{H} \alpha$ ratio throughout the galaxy would introduce systematic overestimations of the $\mathrm{H} \alpha$ flux of up to $40 \%$ in the faintest regions, as well as systematic underestimations of up to $25 \%$ for the brightest regions. The effect is a strong function of the $\mathrm{H} \alpha$ flux, and its magnitude is of the order of the typical uncertainties quoted in narrow-band photometry of star-forming regions in nearby galaxies. While in theory these systematic misestimations should bias a measurement of the slope of the SFL toward shallower values, the magnitude of the effect is ten times smaller than the intrinsic scatter in the SFL and the introduced bias is negligible.

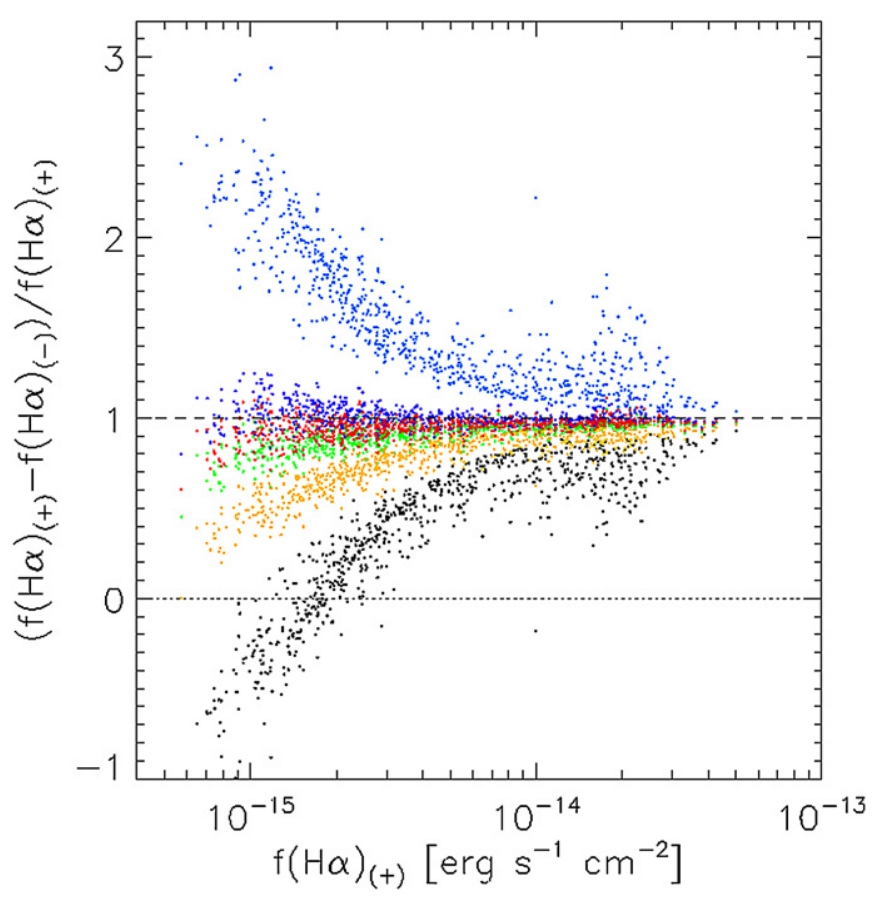

Figure 16. Bias introduced by the misestimation of the strength of the $\mathrm{H} \alpha$ absorption feature or equivalently of the continuum level. Black dots show the fraction of the observed flux that we would observe if the stellar absorption was not considered at all. Red dots show the same fluxes corrected using a constant absorption EW $=-2.4 \AA$ A. Dark blue and green dots correspond to underestimations and overestimations of the continuum by a $10 \%$. Light blue and orange dots correspond to underestimations and overestimations of the continuum by a $50 \%$.

(A color version of this figure is available in the online journal.)

Now let us look at the effects introduced by errors in the continuum subtraction and the estimation of the underlying $\mathrm{H} \alpha$ stellar absorption. When doing narrow-band imaging, the estimated value for the $\mathrm{H} \alpha$ absorption EW is coupled, and impossible to separate from the estimated continuum level. So overestimations of the absorption EW can be thought as underestimations of the subtracted continuum and vice versa. Black dots in Figure 16 show the observed $\mathrm{H} \alpha$ emission flux (before dust extinction correction) versus the fractional difference between the $\mathrm{H} \alpha$ emission and absorption fluxes for all the regions unaffected by AGN contamination. The magnitude of the $\mathrm{H} \alpha$ absorption was measured in the bestfitted stellar continuum spectrum of each region, constructed as described in Section 5.1. The vertical axis in Figure 16 can be interpreted as the fraction of the true flux we would observe if the underlying absorption was not taken out from our measurement. Negative values correspond to regions in which the absorption EW is higher than the emission EW. We measure a fairly constant absorption EW, showing a median of $-2.4 \AA$, and an rms scatter of $0.2 \AA$ between different regions. This supports the approximation of a constant $\mathrm{H} \alpha$ absorption EW on which narrow-band corrections are based. Not taking into account the absorption feature can translate into gross underestimations of the emission line fluxes. For the brightest regions the underestimation can be up to $\sim 50 \%$, and for the faintest regions we could completely miss the presence of nebular emission, and observe pure absorption.

The red dots in Figure 16 show the emission minus absorption fluxes corrected using a constant $\mathrm{H} \alpha$ absorption EW of $-2.4 \AA$. It can be seen that, under the assumption of a constant absorption $\mathrm{EW}$, true fluxes can be recovered with typical uncertainties of 
less than $20 \%$ if the correct value of the median EW is used. Green and blue dots in Figure 16 correspond to the values that would be obtained if the continuum had been overestimated and underestimated by $10 \%$ respectively, or equivalently if the $\mathrm{H} \alpha$ absorption EW had been underestimated by $-0.2 \AA$ and overestimated by $+0.3 \AA$. The orange and light blue dots correspond to continuum misestimations of a $50 \%(-0.8 \AA$, $+2.4 \AA$ ). These offsets are of the same order of magnitude as the typical uncertainties in the continuum subtraction of narrow-band images of nearby galaxies. It can be seen that a systematic misestimations can be introduced to the measured $\mathrm{H} \alpha$ fluxes, especially in the fainter regions. Similarly to the [N II] correction discussed above, this effect is a strong function of the $\mathrm{H} \alpha$ flux and in this case can induce a significant change in the slope of the SFL if the estimated absorption (continuum level) is sufficiently off from the true value. A $10 \%$ error in the continuum level can introduce systematic misestimations of up to $30 \%$, which is small compared to the intrinsic scatter in the SFL, but a $50 \%$ error in the estimation of the continuum can induce misestimations of the measured fluxes that are of the order of the SFL intrinsic scatter, and hence introduce a significant systematic bias to the SFL slope.

We perform a comparison of our spectroscopically measured $\mathrm{H} \alpha$ emission line fluxes to fluxes measured by performing photometry in 4.3 diameter apertures at the positions of each of our fibers on the continuum-subtracted and absorption line corrected narrow-band image used by Calzetti et al. (2005) and Kennicutt et al. (2007). We correct the narrow-band fluxes for [N II] contamination using the correction factors shown in Figure 13, scaled by 0.97 to account for the lower filter transmission at the $\left[\mathrm{N}_{\mathrm{II}}\right]$ lines. Figure 17 shows the comparison. In order to account for differences in flux calibration and photometry aperture effects, we scale the narrow-band fluxes by a factor of 1.25 , given by the mean ratio between the VIRUS$\mathrm{P}$ and narrow-band fluxes for regions with $f(\mathrm{H} \alpha)>10^{-14}$ $\mathrm{erg} \mathrm{s}^{-1} \mathrm{~cm}^{-2}$ (to the right of the dotted line in Figure 15). At high $\mathrm{H} \alpha$ emission fluxes the effects of errors in the continuum subtraction are much smaller than for the fainter regions, so we consider safe to scale the fluxes in order to match the bright end of the distribution, also the magnitude of the scaling factor is of the order of the combined uncertainties in flux calibration.

Narrow-band fluxes presented in Figure 17 should not be affected by previously discussed systematics introduced by [N II] corrections, since we used the spectroscopically measured ratios to correct them. On the other hand, they clearly show a systematic deviation, with narrow-band fluxes being lower than spectroscopic fluxes as we go to fainter regions. This is consistent with an overestimation of the continuum level by $\sim 30 \%$, or equivalently and underestimation of the $\mathrm{H} \alpha$ absorption EW by $-0.6 \AA$, which is well within the uncertainties involved in the continuum subtraction of the narrow-band image (D. Calzetti 2009, private communication). It is important to note that in Kennicutt et al. (2007), the spatially resolved SFL was built by doing photometry on $\mathrm{H} \alpha$ bright star-forming knots (brighter than $3 \times 10^{-15} \mathrm{erg} \mathrm{s}^{-1} \mathrm{~cm}^{-2}$ ), which are less affected by errors in the continuum subtraction than, for example, the interarm regions. Hence we do not expect this effect to significantly affect the slope of the SFL that they measure.

The above comparison stresses a very important point. Although very deep narrow-band imaging can be obtained using present day imagers, low surface brightness photometry of nebular emission in these images is limited by uncertainties in the continuum subtraction and estimation of photospheric absorp-

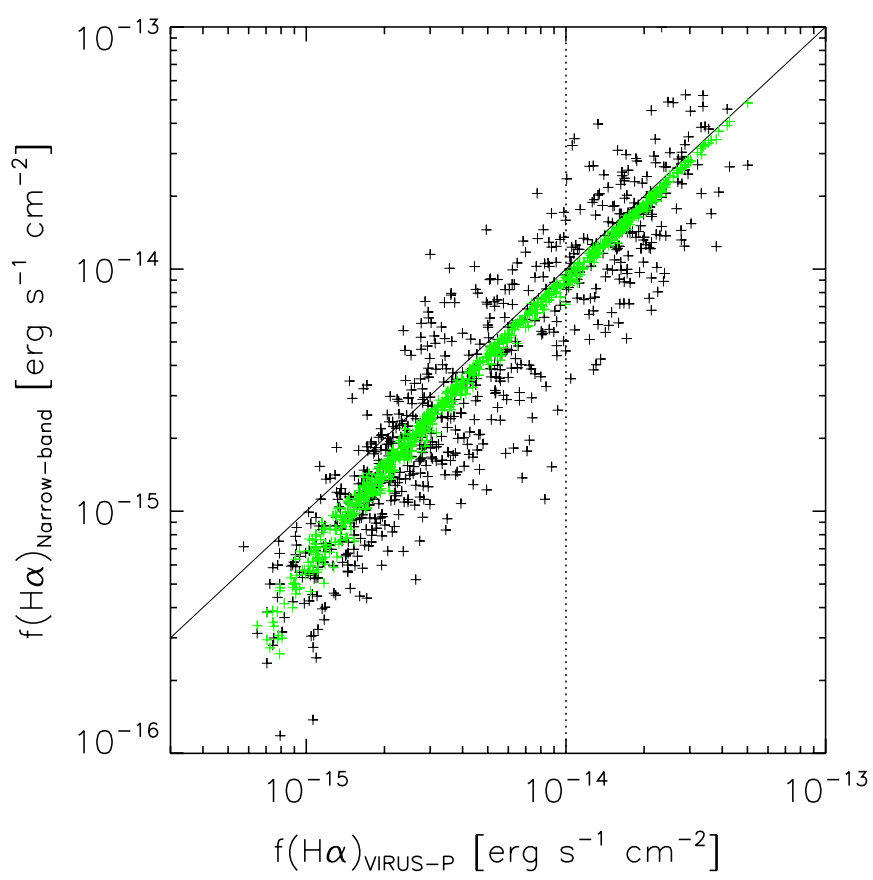

Figure 17. VIRUS-P observed $\mathrm{H} \alpha$ fluxes (before dust extinction correction) vs. $\mathrm{H} \alpha$ fluxes measured in the continuum-subtracted image from Calzetti et al. (2005, black crosses). Data points to the right of the vertical dotted line were used to scale the narrow-band fluxes in order to account for flux calibration and aperture discrepancies. The green crosses show the $\mathrm{H} \alpha$ fluxes that would have been measured by VIRUS-P if the continuum would have been overestimated by a $30 \%$ (see Figure 16).

(A color version of this figure is available in the online journal.)

tion. In this respect, integral field spectroscopy provides us with a less biased way of measuring faint nebular emission in nearby galaxies.

\section{COMPARISON WITH PREVIOUS MEASUREMENTS AND THEORETICAL PREDICTIONS}

In this section, we compare our results to the recent measurements on the spatially resolved SFL in NGC5194 by Kennicutt et al. (2007) and Bigiel et al. (2008), and to the predictions of the theoretical model of the SFL proposed by Krumholz et al. (2009b).

We find an almost complete lack of correlation between the atomic gas surface density and the SFR surface density (Figure 11). This is in good agreement with the observations of both Kennicutt et al. (2007) and Bigiel et al. (2008), and confirms the fact that the SFR is correlated with the molecular gas density, and it is this correlation which drives the power-law part of the total gas SFL. At low gas surface densities $\left(<10 M_{\odot} \mathrm{pc}^{-2}\right)$, the ISM of spiral galaxies stops being mostly molecular, and hence the shape of the total gas SFL is driven by a combination of the molecular gas SFL and the ratio of molecular to atomic hydrogen.

As discussed in Section 1, Kennicutt et al. (2007) find a superlinear slope of 1.37 for the molecular SFL in NGC5194, while Bigiel et al. (2008) measure a slightly sub-linear slope of 0.84 . The first of these measurements is consistent with models in which the SFR is inversely proportional to the gas free-fall time in GMCs and the molecular gas surface density is proportional to the total gas density ( $N=1.5$; Kennicutt 1998 b), while the second is more consistent with models in which the SFR shows a linear correlation with the molecular gas density, product of 


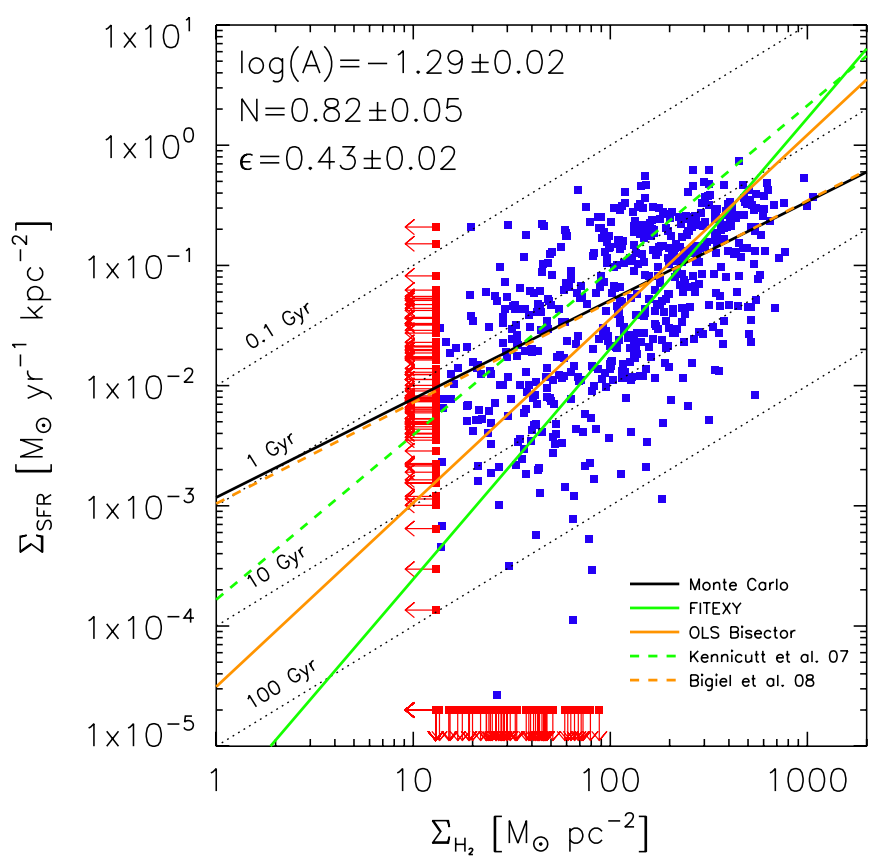

Figure 18. Molecular gas SFL as measured by VIRUS-P. Symbols are the same as in Figure 12. The black solid line shows our best-fitted power-law obtained using the MC method described in Section 9.1. Previous measurements by Kennicutt et al. (2007) and Bigiel et al. (2008) are shown as the green and orange dashed lines, respectively. Also shown are fits to our data (rejecting upper limits) using the FITEXY (solid green line) and OLS bisector (solid orange line) methods.

(A color version of this figure is available in the online journal.)

star formation taking place at a constant efficiency in GMCs. Hence, establishing the slope of the SFL is important in order to distinguish between different physical phenomena that give rise to it.

Figure 18 shows the molecular SFL measured as described in Section 9, together with the best-fitted SFL as measured by Kennicutt et al. (2007) and Bigiel et al. (2008). The results from the latter are adjusted to account for differences in the IMF assumed for calculating $\Sigma_{\mathrm{SFR}}$, and the different $\mathrm{CO}-\mathrm{H}_{2}$ conversion factor used in the calculation of $\Sigma_{\mathrm{H}_{2}}$.

Our best-fitted molecular SFL shows a considerably shallower slope than the one measured by Kennicutt et al. (2007). We consider the source of the disagreement to be a combination of two factors. First, as shown in Section 10, the narrowband $\mathrm{H} \alpha$ fluxes used by Calzetti et al. (2005) and Kennicutt et al. (2007) might be underestimated at the faint end of the flux distribution due to small systematic errors in continuum subtraction, although the effect is small (of the order of the intrinsic scatter in the SFL), and cannot account for the bulk of the difference observed in the SFL slope. The second factor, which we consider to be the main cause behind the disagreement, is the difference in the fitting methods used to adjust a power law to the data. As mentioned in Section 9, Kennicutt et al. (2007) used a FITEXY algorithm to perform a linear regression to the data in logarithmic space, rejecting upper limits in $\Sigma_{\mathrm{H} 2}$ from the fit, and not fitting for the intrinsic scatter in the SFL. The solid green line in Figure 17 shows the result of applying the same procedure to our data. The FITEXY method significantly overpredicts the slope of the SFL $(N=1.9)$, in large part due to the exclusion of the $\Sigma_{\mathrm{H} 2}$ upper limits. These data points, having large error bars in $\Sigma_{\text {gas }}$ and clear detections in $\Sigma_{\mathrm{SFR}}$, have a significant statistical weight in the MC fit because

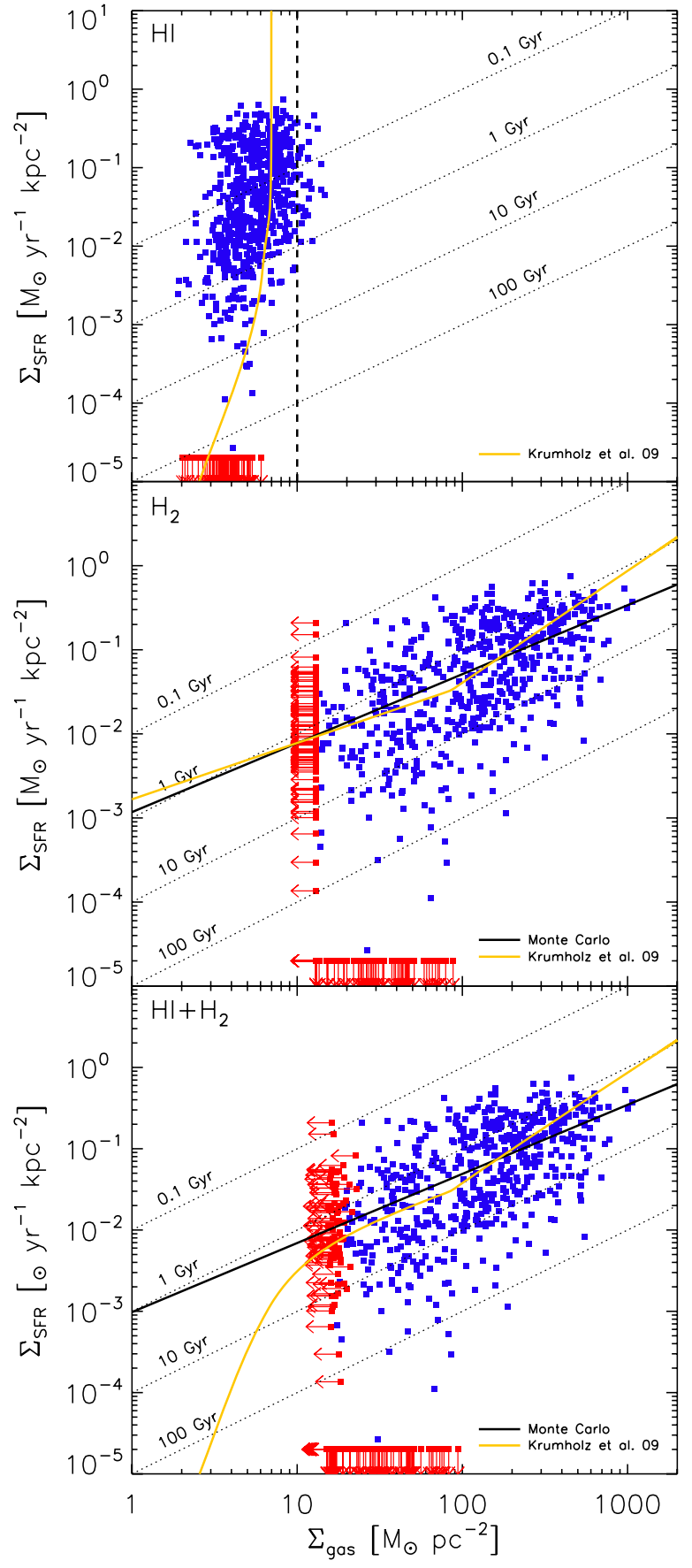

Figure 19. Comparison of the observed SFL for atomic gas (top), molecular gas (center), and total gas (bottom) and the theoretical model proposed by Krumholz et al. (2009b). Symbols are the same as in Figures 11-13. The solid orange line show the Krumholz et al. model for $Z^{\prime}=1.0 / 1.5$ and $c=4$.

(A color version of this figure is available in the online journal.)

of their large number. Another factor promoting the fitting of shallower slopes by our MC method, is the fact that we included the intrinsic dispersion in the SFL as a scatter in $\Sigma_{\text {SFR }}$, hence the fit will tend to equalize the number of data points above and below the power law at any given $\Sigma_{\text {gas }}$. This is a consequence of the expectation for a causal relation between $\Sigma_{\text {gas }}$ and $\Sigma_{\text {SFR }}$, with the SFR being a function of the gas density, and not vice versa.

Kennicutt et al. (2007) provide a table of their measured values for $\Sigma_{\mathrm{SFR}}$ and $\Sigma_{\mathrm{H}_{\mathrm{I}} \mathrm{H}_{2}}$ and their uncertainties, from which 
they recover a slope of $N=1.56$ for the total gas SFL. We apply our MC fitting method to their data, and find best-fitted values of $A=10^{-1.23 \pm 0.03}$ for the amplitude, $\epsilon=0.40 \pm 0.03$ for the intrinsic scatter, and a slope $N=1.03 \pm 0.08$. This shallower slope is a lot closer to our measured value of $N=0.85$, and the rest of the difference can be easily explained by the underestimation of the narrow-band $\mathrm{H} \alpha$ fluxes presented in Figure 17 and differences in the DIG correction. The two independent data sets show excellent agreement in both the normalization and the intrinsic scatter.

On the other hand, we measure a molecular SFL which shows an excellent agreement with Bigiel et al. (2008) both in slope and normalization. The agreement is better than expected, given the differences in the methods used to measure $\Sigma_{\mathrm{SFR}}$ and fitting the SFL. Their SFR measurements are not based on extinctioncorrected hydrogen recombination lines as in Kennicut et al. and this work, but rather on a linear combination of space-based GALEX far-UV and Spitzer MIPS $24 \mu \mathrm{m}$ fluxes. Also, they do not correct their data in order to account for any contribution from the DIG. The fitting method used by Bigiel et al. (2008) is an OLS Bisector, and they also reject non-detections in CO from the fit. The orange solid line shows the result of applying this fitting method to our data. Just as in the case of the FITEXY algorithm, the OLS Bisector yields a significantly higher slope $(N=1.5)$ than the MC fit. The reasons for this are the same as for the FITEXY algorithm, that is, the inclusion of the upper limits in $\Sigma_{\mathrm{H}_{2}}$, and the introduction of the intrinsic scatter in $\Sigma_{\mathrm{SFR}}$ in our method. One possible explanation for the agreement could be the interplay between the lack of DIG correction and the difference in fitting methods. The first will tend to drive the slope to shallower values, while the second will steepen it. The combination of these two effects working in opposite directions might be behind the agreement between Bigiel et al. (2008) and this work.

Although the comparison is hard due to the systematics involved in the different methods, the bottom line is that we measure a slope that is consistent with the scenario proposed by Bigiel et al. (2008) and Leroy et al. (2008), in which star formation takes place at a nearly constant efficiency in GMCs over a large range of environments present in galaxies. This is also in agreement with recent the findings of (Bolatto et al. 2008), who find that extragalactic GMCs in the Local Group, detected on the basis of their $\mathrm{CO}$ emission, exhibit remarkably uniform properties, with a typical mass surface density of roughly $85 M_{\odot} \mathrm{pc}^{-2}$.

Based on these concepts of uniformity of GMC properties, and good correlation between the SFR and the molecular gas density, Krumholz et al. (2009b) proposed a simple theoretical model to explain the observed total gas SFL. In their model, star formation takes place only in molecular gas, and the total gas SFL is determined by three factors. First, the fraction of the gas in molecular form is set by the balance between the formation of $\mathrm{H}_{2}$ in the surface of dust grains and the dissociation of molecules by the far-UV continuum in the Lyman-Werner bands (Krumholz et al. 2008, 2009a). This drives the shape of the total gas SFL in the low-density regime where the ISM is not fully molecular. Second, the SFE inside GMCs is low, and it is set by turbulence-driven feedback processes (Krumholz \& McKee 2005). These are responsible for the power-law behavior of the molecular SFL. Third, GMCs are decoupled from the surrounding ISM when their internal pressure is higher than the external pressure. In this regime, their structure is determined by internal feedback processes, and they show very uniform properties including an almost constant surface density of $85 M_{\odot} \mathrm{pc}^{-2}$ (Bolatto et al. 2008). When the galactic ISM pressure becomes higher than this value, the GMC surface density must increase accordingly in order to maintain pressure balance with the external ISM. This gives rise to a steepening of the slope of the molecular SFL at $\Sigma_{\mathrm{H}_{2}} \geqslant 85 M_{\odot} \mathrm{pc}^{-2}$. In summary, the total gas SFL in the model shows a different behavior in the low-, intermediate-, and high-density regimes. At low densities its behavior is driven by the transition from an atomic to a molecular ISM. Beyond the point at which the ISM becomes almost fully molecular the total gas SFL follows closely the molecular SFL, which shows a steeper slope in the high-density regime driven by the pressure balance between the galactic ISM and GMCs.

Figure 19 shows a comparison of our data and the Krumholz et al. (2009b) model. We have assumed $Z^{\prime}=Z / Z_{\mathrm{MW}}=$ $1.0 / 1.5$, consistently with the DIG correction applied in Section 8 , and a clumpiness factor $c=4$ to account for the effect that the averaging of $\Sigma_{\text {gas }}$ introduces in the molecular fraction in the model. We observe an excellent agreement for both the atomic and molecular gas, as well as for the total gas SFL. The gas density range sampled by our observations, and the scatter in SFL does not allow us to discern between the model and the simple power-law-fitted using the MC method, stressing the need to extend our observations toward more extreme density environments.

\section{SUMMARY AND CONCLUSIONS}

We have performed the first measurement of the spatially resolved SFL in nearby galaxies using integral field spectroscopy. The wide field VIRUS-P spectroscopic map of the central $4.1 \times 4.1 \mathrm{kpc}^{2}$ of NGC 5194, together with the H I $21 \mathrm{~cm}$ map from THINGS, and the CO $J=1-0$ from BIMA SONG were used to measure $\Sigma_{\mathrm{SFR}}, \Sigma_{\mathrm{H}_{\mathrm{I}}}$, and $\Sigma_{\mathrm{H}_{2}}$ for 718 regions $\sim 170 \mathrm{pc}$ in diameter throughout the disk of the galaxy.

In this paper we have presented our method for calculating $\Sigma_{\mathrm{SFR}}$ from the spectroscopically measured $\mathrm{H} \alpha$ emission line fluxes. We have shown that the observed $\mathrm{H} \alpha / \mathrm{H} \beta$ ratio is a good estimator of the nebular dust extinction, at least at the levels of obscuration present in face-on normal spiral galaxies like NGC 5194.

We have also presented a new method for estimating the contribution of the DIG to the $\mathrm{H} \alpha$ emission line flux, which is based on the observed low-ionization line ratio $[\mathrm{S} \mathrm{II}] / \mathrm{H} \alpha$, and the large differences seen in this line ratio between $\mathrm{H}$ II regions and pointings toward the DIG in the MW. The use of line ratios to correct both for dust extinction and the DIG contribution is possible only because of the use of integral field spectroscopy spanning a large wavelength range, which includes all these important emission lines.

One of the main goals of this work is to make use of these clean spectroscopic emission line measurements to study the systematics involved in narrow-band estimations of the $\mathrm{H} \alpha$ emission line flux of nearby galaxies. We showed that proper estimation of the continuum and of the underlying stellar absorption features is crucial in order to get an unbiased estimate of the $\mathrm{H} \alpha$ flux. Errors of the order of $30 \%$ in the estimation of these quantities can introduce systematic misestimations of the $\mathrm{H} \alpha$ emission line flux by up to a factor of 3 in the low surface brightness regime.

We also tested the assumption of a constant $[\mathrm{N} \mathrm{II}] / \mathrm{H} \alpha$ ratio throughout the galaxy, usually used to remove the [N II] doublet contamination from the narrow-band measured fluxes. We found 
that the $[\mathrm{NII}] / \mathrm{H} \alpha$ ratio varies significantly throughout the galaxy, and shows a clear correlation with the $\mathrm{H} \alpha$ flux. The sense of the correlation implies a higher $[\mathrm{NII}] / \mathrm{H} \alpha$ ratio in regions that are fainter in $\mathrm{H} \alpha$ (typically the inter-arm regions of the galaxy), and is consistent with the DIG dominating the nebular spectrum in these zones. Assuming a constant [N II]/ $\mathrm{H} \alpha$ would introduce overestimations of the $\mathrm{H} \alpha$ flux of $\sim 40 \%$ in the inter-arm regions, and underestimations of $\sim 25 \%$ for the brightest star-forming regions in the spiral arms.

Integral field spectroscopy proves to be an extremely powerful tool for mapping the SFR throughout the disks of nearby galaxies, especially with the advent of large field of view IFUs like VIRUS-P. Spatially resolved spectral maps, besides allowing us to measure emission line fluxes in a much more unbiased way than narrow-band imaging, also provide extensive information about the physical conditions throughout the disks of nearby spiral galaxies. The spectra allow the measurement of metallicities, stellar and gas kinematics, stellar populations, and star formation histories across galaxies. In a future study, we will investigate the role that all these other quantities that can be extracted from our data play at setting the SFR.

We found that the SFR surface density shows a lack of correlation with the atomic gas surface density, and a clear correlation with the molecular gas surface density. Hence, the total gas SFL is fully driven by the molecular gas SFL in the density regimes sampled by our observations. The atomic gas surface density is observed to saturate at a value of $\sim 10 M_{\odot} \mathrm{pc}^{-2}$, at which a phase transition between atomic and molecular gas is thought to occur in the ISM.

An MC method for fitting the SFL which is not affected by the systematics involved in performing linear regressions of incomplete data in logarithmic space was presented. Our method fits the intrinsic scatter in the SFL as a free parameter. Applying this method to our data yields slightly sub-linear slopes $N$ of 0.82 and 0.85 for the molecular and total gas SFLs, respectively.

Comparison with previous measurements of the spatially resolved SFL are somewhat challenging because of the different recipes used to estimate $\Sigma_{\text {SFR }}$, and the different fitting procedures used to derive the SFL parameters. The slopes we measured are in disagreement with the results of Kennicutt et al. (2007), who measured a strongly super-linear slope for both the molecular component and the total gas. On the other hand, our results are in very good agreement with the slope measured for the molecular gas SFL in NGC 5194 by Bigiel et al. (2008). Our results are consistent with the scenario recently proposed by Bigiel et al. (2008) and Leroy et al. (2008) of a nearly constant SFE in GMCs, which is almost independent of the molecular gas surface density. The main argument to support this scenario is the observation of a close to linear correlation between the $\Sigma_{\mathrm{SFR}}$ and $\Sigma_{\text {gas }}$ in the density ranges present in the ISM of nearby normal spiral galaxies.

On the other hand our results also show a very good agreement with the more complex scenario recently proposed by Krumholz et al. (2009b), in which the surface density of molecular gas grows with the molecular to atomic fraction at low densities $\left(\Sigma_{\mathrm{H}+\mathrm{H}_{2}} \lesssim 10 M_{\odot} \mathrm{pc}^{-2}\right)$, becomes constant at intermediate densities $\left(10 M_{\odot} \mathrm{pc}^{-2} \lesssim \Sigma_{\mathrm{H}_{\mathrm{I}}+\mathrm{H}_{2}} \lesssim 100 M_{\odot} \mathrm{pc}^{-2}\right)$, and increases linearly with the total gas density in the high-density regime $\left(\Sigma_{\mathrm{H}_{\mathrm{I}} \mathrm{H}_{2}} \gtrsim 100 M_{\odot} \mathrm{pc}^{-2}\right)$. This, combined with an slightly sub-linear efficiency as a function of molecular gas surface density given by the balance between gravitational potential energy and turbulent kinetic energy originated by internal feedback, gives rise to the observed SFL. In their model, the total gas SFL has a super-linear slope $N=1.33$ in the highdensity regime, gets shallower at intermediate densities showing a slope of $N=0.67$, and steepens again at lower densities as the molecular to atomic gas fraction rapidly decreases. Our observations sample the transition between the intermediateand high-density regimes in the model. The intrinsic scatter in the SFL, together with our limited density dynamic range, does not allow us to observe the predicted kink in the SFL directly, but our measured slope of 0.85 is very close to what we expect to measure in a region where we sample both the sub-linear and super-linear parts of the SFL predicted by the Krumholz et al. model. A proper detection of the kink in the SFL predicted by Krumholz et al. (2009b) will require extending the dynamic range to higher gas surface densities.

A major success of the Krumholz et al. (2009b) model is the excellent agreement it shows with the observation with respect to the SFE, or equivalently to the gas depletion timescales. We observe very long depletion timescales of $\tau \approx 2 \mathrm{Gyr}$, in good agreement with previous observations. This time is $\sim 100$ longer than the typical GMC free-fall time. The good agreement between our observations and the Krumholz et al. model implies that this very low efficiency can be easily explained by models in which star formation is self-regulated through turbulence induced by internal mechanical feedback in GMCs.

An important result of this study is the large intrinsic scatter of 0.43 dex observed in both the molecular and total gas SFLs. This translates into a factor of $\sim 3$ scatter in the SFR for regions having the same molecular gas availability, and it may indicate the existence of further parameters that are important in setting the SFR. It is worth mentioning that part of the intrinsic scatter in the SFL must come from the scatter in the SFR-L $(\mathrm{H} \alpha)$ calibration. Charlot \& Longhetti (2001) show that SFRs derived from $\mathrm{H} \alpha$ alone present a large scatter when compared to SFRs derived from full spectral fitting of the stellar populations and nebular emission of a sample of 92 nearby starforming galaxies. Recently, the detection of widespread UV emission beyond the $\mathrm{H} \alpha$ brightness profile cutoff in the outer disks of many nearby galaxies (Gil de Paz et al. 2005; Thilker et al. 2005; Boissier et al. 2007) has raised questions about the proportionality between the $\mathrm{H} \alpha$ emission and the SFR in the low star formation regime. Incomplete sampling of the IMF in low-mass-embedded clusters has been proposed to explain the discrepancy between $\mathrm{H} \alpha$ and UV surface brightness profiles (e.g., Pflamm-Altenburg \& Kroupa 2008). Under this scenario the $\mathrm{H} \alpha$ emission fails to trace star formation in low-mass clusters where statistical fluctuations can translate into a lack of massive ionizing stars, and the SFR-L $(\mathrm{H} \alpha)$ becomes nonlinear in the low star formation regime (Pflamm-Altenburg et al. 2007), which might enhance the downward scatter in our SFL measurements. This issue is beyond the scope of the current paper, but we intend to investigate the implications of applying nonlinear SFR-L $(\mathrm{H} \alpha)$ to our data in future works.

In this paper, we have established the method for studying the spatially resolved SFL using wide integral field spectroscopy, and have set new constrains on important quantities such as the slope, normalization, and intrinsic scatter of the SFL. As mentioned in Section 1, these data forms part of an undergoing large-scale IFU survey of nearby galaxies. VENGA will map the disks of $\sim 20$ nearby spiral galaxies to radius much larger than those sampled by the data presented here. In the future, we will extend this type of study to a larger set of galaxies spanning a range in Hubble types, metallicities, and star formation activities. This will help us to sample a larger dynamical range 
in gas surface densities. The later requires the observation of much denser environments, such as those present in starburst galaxies, to extend the observed SFL to higher densities. Deeper $\mathrm{CO}$ observations that map the molecular gas out to large radii will be necessary to extend the sampled range to lower densities. This is of great importance, since a proper characterization of the shape of the total gas SFL is necessary in order to distinguish between different star formation models.

We thank Phillip McQueen and Gary Hill for designing and constructing VIRUS-P, and for their advice on the use of the instrument. We also acknowledge David Doss and the staff at McDonald Observatory for their invaluable help during the observations. Tables of the WHAM DIG line ratios were kindly provided by George J. Madsen. We thank Daniela Calzetti for some very useful discussions, and for providing the narrowband $\mathrm{Pa} \alpha$ and $\mathrm{H} \alpha$ images used in her work. This study has been possible thanks to the financial support of Sigma Xi, The Scientific Research Society. The construction of VIRUS-P was possible thanks to the generous support of the Cynthia \& George Mitchell Foundation. N.J.E. and A.H. were supported in part by NSF Grant AST-0607193. This research has made use of the NASA/IPAC Extragalactic Database (NED) which is operated by the Jet Propulsion Laboratory, California Institute of Technology, under contract with the National Aeronautics and Space Administration, and of NASA's Astrophysics Data System Bibliographic Services. Finally we thank the referee for the helpful comments that helped to improve the quality of this work.

\section{REFERENCES}

Allende Prieto, C., Lambert, D. L., \& Asplund, M. 2001, ApJ, 556, L63 Beers, T. C., Flynn, K., \& Gebhardt, K. 1990, AJ, 100, 32

Bigiel, F., Leroy, A., Walter, F., Brinks, E., de Blok, W. J. G., Madore, B., \& Thornley, M. D. 2008, AJ, 136, 2846

Blitz, L., Fukui, Y., Kawamura, A., Leroy, A., Mizuno, N., \& Rosolowsky, E. 2007, in Protostars and Planets V, ed. B. Reipurth, D. Jewitt, \& K. Keil (Tucson, AZ: Univ. Arizona Press), 81

Bloemen, J. B. G. M., et al. 1986, A\&A, 154, 25

Boissier, S., et al. 2007, ApJS, 173, 524

Bolatto, A. D., Leroy, A. K., Rosolowsky, E., Walter, F., \& Blitz, L. 2008, ApJ, 686, 948

Bradley, L. D., Kaiser, M. E., \& Baan, W. A. 2004, ApJ, 603, 463

Bresolin, F., Garnett, D. R., \& Kennicutt, R. C., Jr. 2004, ApJ, 615, 228

Calzetti, D., et al. 2005, ApJ, 633, 871

Charlot, S., \& Longhetti, M. 2001, MNRAS, 323, 887

Crane, P. C., \& van der Hulst, J. M. 1992, AJ, 103, 1146

Deharveng, L., Peña, M., Caplan, J., \& Costero, R. 2000, MNRAS, 311, 329

Dolphin, A. E., et al. 2001, MNRAS, 324, 249

Dove, J. B., Shull, J. M., \& Ferrara, A. 2000, ApJ, 531, 846

Evans, N. J., et al. 2009, ApJS, 181, 321
Ferguson, A. M. N., Wyse, R. F. G., Gallagher, J. S., III, \& Hunter, D. A. 1996, AJ, 111, 2265

Gil de Paz, A., et al. 2005, ApJ, 627, L29

Greenawalt, B., Walterbos, R. A. M., Thilker, D., \& Hoopes, C. G. 1998, ApJ, 506,135

Grevesse, N., Noels, A., \& Sauval, A. J. 1996, Cosmic Abundances, 99, 117

Haffner, L. M., et al. 2009, Rev. Mod. Phys., 81, 969

Helfer, T. T., Thornley, M. D., Regan, M. W., Wong, T., Sheth, K., Vogel, S. N., Blitz, L., \& Bock, D. C.-J. 2003, ApJS, 145, 259

Hill, G. J., et al. 2008, Proc. SPIE, 7014, 701470

Hoopes, C. G., \& Walterbos, R. A. M. 2003, ApJ, 586, 902

Hoopes, C. G., Walterbos, R. A. M., \& Greenwalt, B. E. 1996, AJ, 112, 1429

Isobe, T., Feigelson, E. D., Akritas, M. G., \& Babu, G. J. 1990, ApJ, 364, 104

Kennicutt, R. C., Jr. 1998a, ARA\&A, 36, 189

Kennicutt, R. C., Jr. 1998b, ApJ, 498, 541

Kennicutt, R. C., Jr., et al. 2007, ApJ, 671, 333

Kewley, L. J., Dopita, M. A., Sutherland, R. S., Heisler, C. A., \& Trevena, J. 2001, ApJ, 556, 121

Krumholz, M. R., \& McKee, C. F. 2005, ApJ, 630, 250

Krumholz, M. R., McKee, C. F., \& Tumlinson, J. 2008, ApJ, 689, 865

Krumholz, M. R., McKee, C. F., \& Tumlinson, J. 2009a, ApJ, 693, 216

Krumholz, M. R., McKee, C. F., \& Tumlinson, J. 2009b, ApJ, 699, 850

Krumholz, M. R., \& Tan, J. C. 2007, ApJ, 654, 304

Leroy, A. K., Walter, F., Brinks, E., Bigiel, F., de Blok, W. J. G., Madore, B., \& Thornley, M. D. 2008, AJ, 136, 2782

Madsen, G. J., Reynolds, R. J., \& Haffner, L. M. 2006, ApJ, 652, 401

Mathis, J. S. 2000, ApJ, 544, 347

McKee, C. F. 1999, in NATO ASI Conf. Proc. 540, The Origin of Stars and Planetary Systems, ed. C. J. Lada \& N. D. Kylafis (Dordrecht: Kluwer), 29

Mutchler, M., et al. 2005, BAAS, 37, 452

Oke, J. B. 1990, AJ, 99, 1621

Oey, M. S., et al. 2007, ApJ, 661, 801

Osterbrock, D. E., \& Ferland, G. J. 2006, Astrophysics of Gaseous Nebulae and Active Galactic Nuclei (2nd. ed.; Sausalito, CA: Univ. Science Books)

Pei, Y. C. 1992, ApJ, 395, 130

Pflamm-Altenburg, J., Weidner, C., \& Kroupa, P. 2007, ApJ, 671, 1550

Pflamm-Altenburg, J., \& Kroupa, P. 2008, Nature, 455, 641

Press, W. H., Flannery, B. P., Teukolsky, S. A., \& Vetterling, W. T. 1989, Numerical Recipes in Pascal. The Art of Scientific Computing (Cambridge: University Press)

Reynolds, R. J. 1985, ApJ, 294, 256

Schlegel, D. J., Finkbeiner, D. P., \& Davis, M. 1998, ApJ, 500, 525

Schmidt, M. 1959, ApJ, 129, 243

Schuster, K. F., Kramer, C., Hitschfeld, M., Garcia-Burillo, S., \& Mookerjea, B. 2007, A\&A, 461, 143

Scoville, N. Z., Polletta, M., Ewald, S., Stolovy, S. R., Thompson, R., \& Rieke, M. 2001, AJ, 122, 3017

Shaver, P. A., McGee, R. X., Newton, L. M., Danks, A. C., \& Pottasch, S. R. 1983, MNRAS, 204, 53

Terashima, Y., \& Wilson, A. S. 2001, ApJ, 560, 139

Thilker, D. A., Walterbos, R. A. M., Braun, R., \& Hoopes, C. G. 2002, AJ, 124, 3118

Thilker, D. A., et al. 2005, ApJ, 619, L79

Tully, R. B. 1974, ApJS, 27, 437

Valdes, F., Gupta, R., Rose, J. A., Singh, H. P., \& Bell, D. J. 2004, ApJS, 152, 251

Veilleux, S., \& Osterbrock, D. E. 1987, ApJS, 63, 295

Walter, F., Brinks, E., de Blok, W. J. G., Bigiel, F., Kennicutt, R. C., Thornley, M. D., \& Leroy, A. 2008, AJ, 136, 2563

Wong, T., \& Blitz, L. 2002, ApJ, 569, 157 